\title{
ENERGY
}

\section{Kinetics of Hot-Gas Desulfurization Sorbents for Transport Reactors}

Annual Technical Progress Report for the Period October 1, 1999 to September 30, 2000

By

K. C. Kwon

Tel: (334) 727-8976, Fax: (334) 724-4188

January 2001

Work Performed Under Contract No

DE-FG26-98FT40145

For

U.S. Department of Energy

National Energy Technology Laboratory

Pittsburgh, PA 15236-0940

By

Tuskegee University

Tuskegee, Alabama 36088 
Kinetics of Hot-Gas Desulfurization Sorbents for Transport Reactors

Annual Technical Progress Report

for the Period October 1, 1999 to September 30, 2000

K. C. Kwon

Tel: (334) 727-8976, Fax: (334) 724-4188

January 2001

Work Performed Under Contract No.: DE-FG26-98FT40145

For

U.S. Department of Energy

Office of Fossil Energy

National Energy Technology Laboratory

P.O. Box 10940

Pittsburgh, PA 15236-0940

By

Tuskegee University

Tuskegee, Alabama 36088 


\section{DISCLAIMER}

This report was prepared as an account of work sponsored by an agency of the United States Government. Neither the United States Government nor any agency thereof, nor any of their employees, makes any warranty, express or implied, or assume any legal liability or responsibility for the accuracy, completeness, or usefulness of any information, apparatus, product, or process disclosed, or represents that its use would not infringe privately owned rights. Reference herein to any specific commercial product, process, or service by trade name, trademark, manufacturer, or otherwise does not necessarily constitute or imply its endorsement, recommendation, or favoring by the United States Government or any agency thereof. The views and opinions of authors expressed herein do not necessarily state or reflect those of the United States Government or any agency thereof. 


\section{CONTENTS}

Page

DISCLAIMER

LIST OF TABLES v v

LIST OF FIGURES $\quad$ vi

SUMMARY

$\begin{array}{ll}\text { INTRODUCTION } & 1\end{array}$

EXPERIMENTAL SETUPS AND PROCEDURES 2

$\begin{array}{lr}\text { THEORY } & 9\end{array}$

$\begin{array}{lr}\text { RESULTS AND DISCUSSION } & 10\end{array}$

Effects of Temperature on Initial Dynamic Absorption of $\mathrm{H}_{2} \mathrm{~S} \quad 12$

Effects of Sorbent amounts on Initial Dynamic Absorption of $\mathrm{H}_{2} \mathrm{~S} \quad 14$

Effects of Concentration of $\mathrm{H}_{2} \mathrm{~S}$ on Initial Dynamic Absorption of $\mathrm{H}_{2} \mathrm{~S} \quad 17$

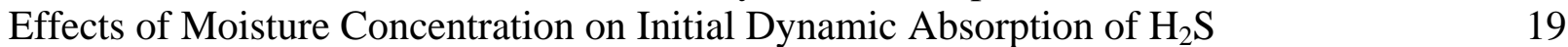

Effects of Hydrogen Concentration on Initial Dynamic Absorption of $\mathrm{H}_{2} \mathrm{~S}$

$\begin{array}{ll}\text { CONCLUSIONS } & 21\end{array}$

$\begin{array}{ll}\text { REFERENCES } & 22\end{array}$

$\begin{array}{lr}\text { PUBLICATIONS } & 23\end{array}$ 


\section{LIST OF TABLES}

Table

Page

1 Experimental conditions for the reaction of hydrogen sulfide with the sorbents.

2 Properties of the AHI-5 sorbent from the Research Triangle Institute (RTI).

4 Absorption of hydrogen sulfide into AHI-5 sorbent in the presence of hydrogen and moisture at various absorption conditions.

5 Surface reaction rate constants and intraparticle diffusivity values calculated with initial absorption data and the reaction model. 


\section{LIST OF FIGURES}

Figure

Page

$1 \quad$ A differential reactor assembly

2 Effects of reaction duration on absorption of 12000-ppm $\mathrm{H}_{2} \mathrm{~S}$ on 0.01-g AHI-5 sorbent with $10-\mathrm{v} \%$ hydrogen and $10-\mathrm{v} \%$ moisture at $450^{\circ} \mathrm{C}$ and the space time $0.076 \mathrm{~s}$.

3 Effects of temperature on absorption of 12000-ppm $\mathrm{H}_{2} \mathrm{~S}$ on 0.01-g AHI-5 sorbent with $10-\mathrm{v} \%$ hydrogen and 10-v\% moisture.

4 Effects of temperature on absorption of 12000-ppm $\mathrm{H}_{2} \mathrm{~S}$ on 0.01-g AHI-5 sorbent with $10-\mathrm{v} \%$ hydrogen and $10-\mathrm{v} \%$ moisture, using the reaction model.

5 Effects of temperature on surface reaction rate constant and intraparticle diffusivity in the absorption of 12000-ppm $\mathrm{H}_{2} \mathrm{~S}$ into AHI-5 sorbent with 10-v\% $\mathrm{H}_{2}$ and $10-\mathrm{v} \%$ moisture.

6 Effects of temperature on surface reaction rate constant in the absorption of 12000-ppm $\mathrm{H}_{2} \mathrm{~S}$ into AHI-5 sorbent with 10-v\% $\mathrm{H}_{2}$ and 10-v\% moisture, using the Arrhenius equation.

7 Effects of sorbent amount on absorption of 18000-ppm $\mathrm{H}_{2} \mathrm{~S}$ on AHI-5 sorbent with $10-\mathrm{v} \%$ hydrogen and $10-\mathrm{v} \%$ moisture at $450^{\circ} \mathrm{C}$ and the space time $0.076 \mathrm{~s}$.

8 Effects of sorbent amount on absorption of 18000-ppm $\mathrm{H}_{2} \mathrm{~S}$ on AHI-5 sorbent with $10-\mathrm{v} \%$ hydrogen and $10-\mathrm{v} \%$ moisture at $450^{\circ} \mathrm{C}$ and the space time $0.076 \mathrm{~s}$, using the reaction model.

9 Effects of sorbent amount on surface reaction rate constant and intraparticle diffusivity in the absorption of 18000-ppm $\mathrm{H}_{2} \mathrm{~S}$ into AHI-5 sorbent with 10-v\% $\mathrm{H}_{2}$ and $10-\mathrm{v} \%$ moisture at $450^{\circ} \mathrm{C}$.

10 Effects of $\mathrm{H}_{2} \mathrm{~S}$ concentration on absorption of $\mathrm{H}_{2} \mathrm{~S}$ on $0.01-\mathrm{g}$ AHI-5 sorbent with $10-\mathrm{v} \%$ hydrogen and $10-\mathrm{v} \%$ moisture at $450^{\circ} \mathrm{C}$ and the space time $0.076 \mathrm{~s}$.

11 Effects of $\mathrm{H}_{2} \mathrm{~S}$ concentration on absorption of $\mathrm{H}_{2} \mathrm{~S}$ on $0.01-\mathrm{g}$ AHI-5 sorbent with $10-\mathrm{v} \%$ hydrogen and $10-\mathrm{v} \%$ moisture at $450^{\circ} \mathrm{C}$ and the space time $0.076 \mathrm{~s}$, using the reaction model.

12 Effects of $\mathrm{H}_{2} \mathrm{~S}$ concentration on surface reaction rate constant and intraparticle diffusivity in the absorption of $\mathrm{H}_{2} \mathrm{~S}$ into $0.01-\mathrm{g}$ AHI-5 sorbent with $10-\mathrm{v} \% \mathrm{H}_{2}$ and $10-\mathrm{v} \%$ moisture at $450^{\circ} \mathrm{C}$. 


\section{LIST OF FIGURES Continued-1}

13 Effects of moisture concentration on absorption of 18000-ppm $\mathrm{H}_{2} \mathrm{~S}$ on 0.01-g AHI-5 sorbent with $10-\mathrm{v} \%$ hydrogen at $450^{\circ} \mathrm{C}$ and the space time $0.076 \mathrm{~s}$.

14 Effects of moisture concentration on absorption of 18000-ppm $\mathrm{H}_{2} \mathrm{~S}$ on 0.01-g AHI-5 sorbent with $10-\mathrm{v} \%$ hydrogen at $450^{\circ} \mathrm{C}$ and the space time $0.076 \mathrm{~s}$, using the reaction model.

15 Effects of moisture concentration on surface reaction rate constant and intraparticle diffusivity in the absorption of $\mathrm{H}_{2} \mathrm{~S}$ into $0.01-\mathrm{g}$ AHI-5 sorbent with $10-\mathrm{v} \% \mathrm{H}_{2}$ at $450^{\circ} \mathrm{C}$.

16 Effects of hydrogen concentration on absorption of 18000-ppm $\mathrm{H}_{2} \mathrm{~S}$ on $0.01-\mathrm{g}$ AHI-5 sorbent with $10-\mathrm{v} \%$ moisture at $450^{\circ} \mathrm{C}$ and the space time $0.076 \mathrm{~s}$.

17 Effects of hydrogen concentration on absorption of $18000-\mathrm{ppm}_{2} \mathrm{~S}$ on $0.01-\mathrm{g}$ AHI-5 sorbent with $10-\mathrm{v} \%$ moisture at $450^{\circ} \mathrm{C}$ and the space time $0.076 \mathrm{~s}$, using the reaction model.

18 Effects of hydrogen concentration on surface reaction rate constant and intraparticle diffusivity in the absorption of $\mathrm{H}_{2} \mathrm{~S}$ into 0.01 -g AHI-5 sorbent with $10-\mathrm{v} \%$ moisture at $450^{\circ} \mathrm{C}$. 


\section{SUMMARY}

Hot-gas desulfurization for the integrated gasification combined cycle (IGCC) process has been investigated by many researchers to remove effectively hydrogen sulfide with various metal oxide sorbents at elevated temperatures. Various metal oxide sorbents are formulated with metal oxides such as $\mathrm{Fe}, \mathrm{Co}, \mathrm{Zn}$, and $\mathrm{Ti}$. Initial reaction kinetics of formulated sorbents with hydrogen sulfide is studied in the presence of various amounts of moisture and hydrogen at various reaction temperatures. The objectives of this research are to study initial reaction kinetics for a sorbent-hydrogen sulfide heterogeneous reaction system, to investigate effects of concentrations of hydrogen sulfide, hydrogen, and moisture on dynamic absorption of $\mathrm{H}_{2} \mathrm{~S}$ into sorbents, and to evaluate effects of temperature and sorbent amounts on dynamic absorption of $\mathrm{H}_{2} \mathrm{~S}$ into sorbents. Experimental data on initial reaction kinetics of hydrogen sulfide with metal oxide sorbents were obtained with a $0.83-\mathrm{cm}^{3}$ differential reactor. In this report, the reactivity of AHI-5 was examined. This sorbent was obtained from the Research Triangle Institute (RTI). The sorbent in the form of $70 \mu \mathrm{m}$ particles are reacted with $9000-18000$ ppm hydrogen sulfide at $350-500^{\circ} \mathrm{C}$. The range of space time of reaction gas mixtures is $0.071-0.088 \mathrm{~s}$. The range of reaction duration is $4-10800 \mathrm{~s}$.

\section{INTRODUCTION}

Coal is the most plentiful energy resource in the United States and continues to be the major fuel utilized by electrical power plants. The coal resources will become attractive for chemical feedstocks as well as for a source of liquid and gaseous fuels as petroleum resources become continually depleted.

Coal is a very complex and heterogeneous material. Effective utilization of coal requires an understanding of the formation, structure, and purification of coal. Raw coal consists of minerals and organic matter. The minerals include primarily pyrites, silicate (quartz, clays) and various minor minerals, whereas organic matter is composed of carbon, oxygen, hydrogen, nitrogen and sulfur. Sulfur in coal is present in organic, pyritic and sulfate forms. Although the sulfur occurring in the form of relatively coarse pyrite particles is removed largely by density separation, very finely disseminated pyrite and organic sulfur cannot be removed in this way. Sulfur products from combustion not only pollute the environment, but are also detrimental to combustion systems because of the corrosive action of their combustion products. In the gasification process, coal is reacted with steam and oxygen to produce a medium to high calorific value fuel gas. The fuel gas can be subsequently combusted in a gas/steam turbine combined cycle (IGCC). These IGCC-based power generation options offer much higher thermodynamic cycle efficiencies that promise to remove all forms of pollutants released from a coal conversion process through lower fuel consumption.

The removal of hydrogen sulfide from hot coal gas produced in the IGCC- based system by metal oxide sorbents is required to protect downstream combustion turbines from being corroded with sulfur compounds. The removal of sulfur compounds from coal gas products is investigated by using various metal oxide sorbents and membrane separation methods. Current 
investigations show that the removal of sulfur with metal oxide sorbents appears to deliver the most promising results. The main requirements of these metal oxide sorbents are durability and a high sulfur-loading capacity during absorption-regeneration cycles.

\section{EXPERIMENTAL SETUPS AND PROCEDURES}

A differential micro reactor was fabricated with a titanium grade- 2 tube. The volume of reactor is $0.83 \mathrm{~cm}^{3}$. The RTI AHI-5 sorbent in the form of $70-\mu \mathrm{m}$ spherical particles was examined. These sorbent particles were reacted with simulated coal gases containing hydrogen sulfide in the $0.83 \mathrm{~cm}^{3}$ titanium Grade-2 differential micro reactor for $4-10800$ seconds at 350 $500^{\circ} \mathrm{C}$. Absorption amounts of hydrogen sulfide into the sorbent were analyzed with the LECO Sulfur Analyzer. The range of space (residence) time of the reaction gas mixture in the reactor was $0.071-0.088 \mathrm{~s}$ under the reaction conditions.

\section{Figure 1. A Differential Reactor Assembly}

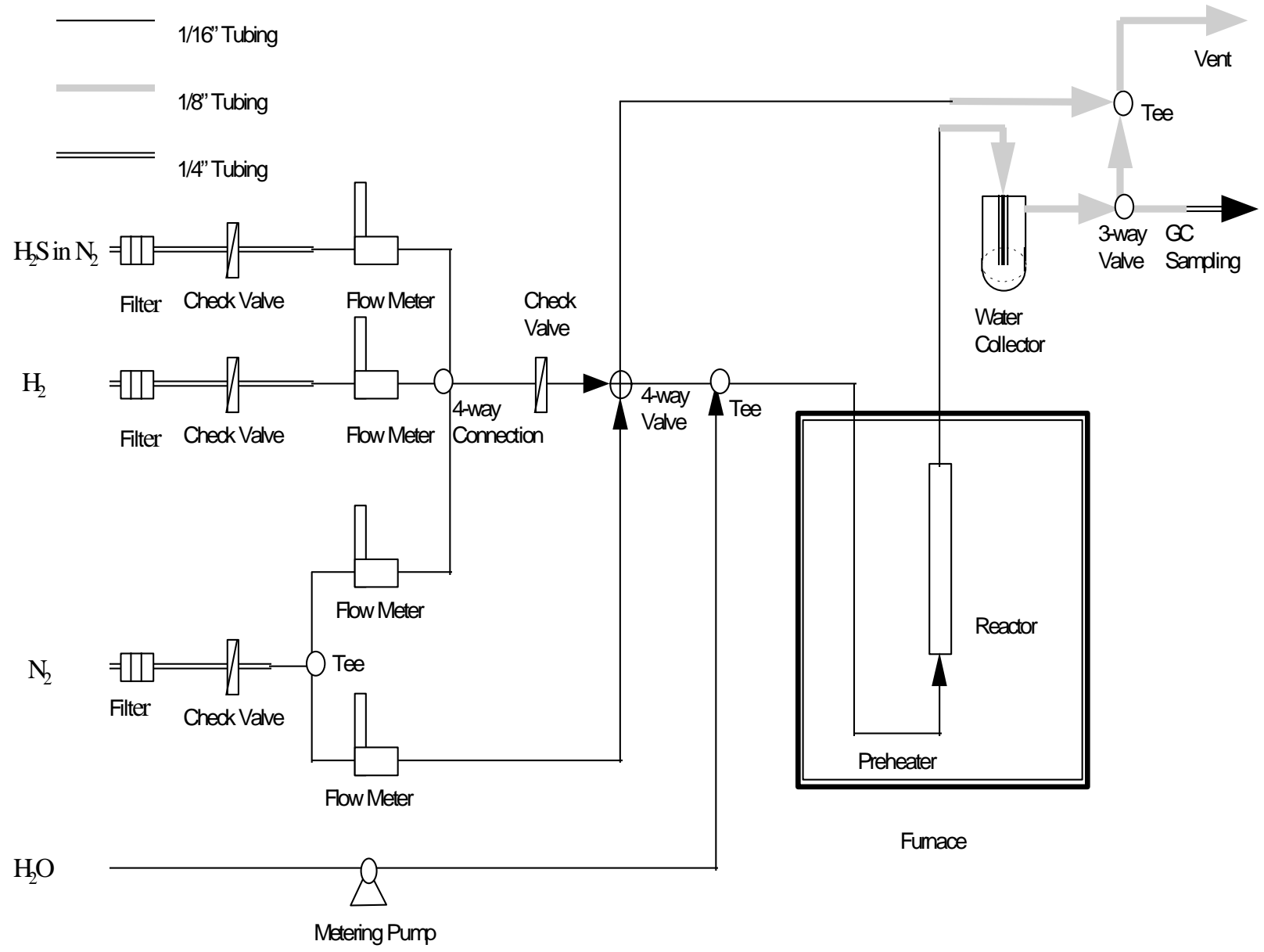


A differential reactor assembly mainly consists of four mass flow meters for gases, one differential reactor, one preheater, one high pressure liquid pump for water, one four-way switch valve, one muffle furnace, three filters for gases, four check valves, and one water collection bottle (see Figure 1 ). The differential reactor is fabricated with one 5 - $\mathrm{cm}$-long $1 / 4$ inch titanium grade-2 tubing and two $1 / 4$ " external column end fittings. The preheater is made of $1 / 16$ " Hastelloy $C$ tubing. The reactor was loaded with $70 \mu \mathrm{m}$ sorbent particles. The reactor, loaded with the sorbent particles, was placed inside the muffle furnace to be heated at a desired temperature. Nitrogen was introduced into the sorbent-loaded reactor during preheating the reactor. When the temperature of the reactor was raised at the desired temperature, a simulated coal gas mixture, containing $\mathrm{H}_{2} \mathrm{~S}$, was introduced into the reactor for desired reaction duration, by switching nitrogen with the simulated coal gas mixture. A reaction was terminated at desired reaction duration, and then absorption of $\mathrm{H}_{2} \mathrm{~S}$ into the sorbent was analyzed with the LECO sulfur analyzer. These raw experimental data were shown in Table 3. The typical reaction conditions are shown in Table 1. The properties of the sorbents are shown in Table 2.

Table 1. Experimental conditions for the reaction of hydrogen sulfide with the sorbents.

$\begin{array}{ll}\text { Reactor Volume, } \mathrm{cm}^{3}: & 0.83 \\ \text { Temperature, }{ }^{\circ} \mathrm{C}: & 350-500 \\ \text { Reaction Pressure, psia } & 40.7 \\ \text { Reaction Time, s: } & 4-10800 \\ \text { Space Time under the absorption conditions, s: } & 0.071-0.088 \\ \text { Mean Particle Size, } \mu \mathrm{m} & 70 \\ \text { Amount of Sorbent, g } & 0.005-0.02 \\ \text { Gas Flow Rate, cc/min at room temperature and 1 atm } & 750 \\ \text { Hydrogen, vol \% } & 0-27 \\ \text { Moisture, vol \%: } & 5-20 \\ \text { Concentration of } \mathrm{H}_{2} \mathrm{~S}, \mathrm{ppm} & 9000-18000 \\ \text { Nitrogen, vol \% } & \text { Remainder }\end{array}$

Table 2. Properties of the sorbents from the Research Triangle Institute (RTI).

Sorbent

BET Area, $\mathrm{m}^{2} / \mathrm{g}$

Avg. Pore Diameter, $\AA$, based on volume

Density, $\mathrm{g} / \mathrm{cm}^{3}$

Pore Volume, $\mathrm{cm}^{3} / \mathrm{g}$

Mean Particle Size, $\mu \mathrm{m}$

Composition
AHI-5

86

500

2.077

0.224

70

$75 \mathrm{w} \% \mathrm{Fe}_{2} \mathrm{O}_{3}$

$15 \mathrm{w} \% \mathrm{ZnO}$

$10 \mathrm{w} \%$ Inerts 
Table 3. Absorption of hydrogen sulfide into $\mathrm{AHI}-5$ sorbent in the presence of hydrogen and moisture at various absorption conditions.

\begin{tabular}{|c|c|c|c|c|c|c|c|c|c|c|}
\hline Run \# & $\begin{array}{l}\text { Sorbent } \\
\text { Amount, } g\end{array}$ & $\begin{array}{l}\text { Reaction } \\
\text { Duration, } s\end{array}$ & $\begin{array}{c}\text { Sulfur } \\
\text { Absorption, w\% }\end{array}$ & $\begin{array}{l}\text { Reaction } \\
\text { Temperature, }{ }^{\circ} \mathrm{C}\end{array}$ & $\begin{array}{c}\text { Hydrogen } \\
\text { Sulfide, ppm }\end{array}$ & $\begin{array}{l}\text { Hydrogen } \\
\text { v\% }\end{array}$ & $\begin{array}{l}\text { Moisture } \\
\text { v\% }\end{array}$ & $\begin{array}{l}\text { Space } \\
\text { Time, s }\end{array}$ & $\begin{array}{l}\text { Reaction Rate } \\
\text { Constant, } \mathrm{cm} / \mathrm{s}\end{array}$ & $\begin{array}{l}\text { Intraparticle } \\
\text { Diffusivity, } \mathrm{cm}^{2} / \mathrm{s}\end{array}$ \\
\hline 1075 & 0.0108 & 4.5 & 0.53 & 450 & 12000 & 10 & 10 & 0.076 & 1.2034 & 0.002868 \\
\hline 1076 & 0.0101 & 8.3 & 4.002 & & & & & & & \\
\hline 1077 & 0.0108 & 12.27 & 7.703 & & & & & & & \\
\hline 1078 & 0.0103 & 16.11 & 9.123 & & & & & & & \\
\hline 1079 & 0.0105 & 20.29 & 11.89 & & & & & & & \\
\hline 1080 & 0.0105 & 25.28 & 14.02 & & & & & & & \\
\hline 1081 & 0.0102 & 30.41 & 16.88 & & & & & & & \\
\hline 1082 & 0.0108 & 60.16 & 24.64 & & & & & & & \\
\hline 1083 & 0.0105 & 180.16 & 29.02 & & & & & & & \\
\hline 1093 & 0.0104 & 4.31 & 0.6983 & 400 & 12000 & 10 & 10 & 0.081 & 0.8832 & 0.001731 \\
\hline 1084 & 0.0107 & 3.94 & 1.443 & & & & & & & \\
\hline 1085 & 0.0104 & 8.24 & 2.211 & & & & & & & \\
\hline 1086 & 0.0109 & 12.23 & 4.436 & & & & & & & \\
\hline 1087 & 0.0104 & 16.13 & 7.772 & & & & & & & \\
\hline 1088 & 0.0105 & 20.3 & 7.492 & & & & & & & \\
\hline 1089 & 0.0105 & 25.1 & 12.57 & & & & & & & \\
\hline 1090 & 0.0108 & 30.06 & 7.671 & & & & & & & \\
\hline 1091 & 0.0107 & 60.07 & 22.3 & & & & & & & \\
\hline 1092 & 0.0106 & 180.41 & 26.8 & & & & & & & \\
\hline 1094 & 0.0109 & 4.37 & 1.075 & 350 & 12000 & 10 & 10 & 0.088 & 0.1591 & 0.000222 \\
\hline 1095 & 0.0108 & 8.41 & 1.218 & & & & & & & \\
\hline 1096 & 0.0107 & 12.1 & 1.348 & & & & & & & \\
\hline 1097 & 0.0106 & 16.21 & 1.687 & & & & & & & \\
\hline 1098 & 0.0107 & 20.37 & 1.579 & & & & & & & \\
\hline 1099 & 0.0106 & 25.42 & 2.577 & & & & & & & \\
\hline 1100 & 0.0104 & 30.27 & 2.508 & & & & & & & \\
\hline 1101 & 0.0105 & 60.27 & 5.764 & & & & & & & \\
\hline 1102 & 0.01 & 180.17 & 16.41 & & & & & & & \\
\hline 1122 & 0.0102 & 360.2 & 22.73 & & & & & & & \\
\hline 1104 & 0.0106 & 4.28 & 1.688 & 500 & 12000 & 10 & 10 & 0.071 & 1.7153 & 0.001953 \\
\hline 1105 & 0.0102 & 8.34 & 4.623 & & & & & & & \\
\hline 1106 & 0.0105 & 12.46 & 9.144 & & & & & & & \\
\hline 1107 & 0.0108 & 16.26 & 12.2 & & & & & & & \\
\hline 1108 & 0.0103 & 20.28 & 15.19 & & & & & & & \\
\hline 1109 & 0.0106 & 25.34 & 16.04 & & & & & & & \\
\hline 1110 & 0.0104 & 30.38 & 17.09 & & & & & & & \\
\hline 1111 & 0.0108 & 60.29 & 22.28 & & & & & & & \\
\hline 1112 & 0.0108 & 180.17 & 27.5 & & & & & & & \\
\hline 1113 & 0.0104 & 4.44 & 3.168 & 450 & 18000 & 10 & 10 & 0.076 & 0.9374 & 0.002317 \\
\hline 1114 & 0.0102 & 8.15 & 8.023 & & & & & & & \\
\hline
\end{tabular}


Table 3. continued-1

\begin{tabular}{|c|c|c|c|c|c|c|c|c|c|c|}
\hline Run\# & $\begin{array}{l}\text { Sorbent } \\
\text { Amount, } g\end{array}$ & $\begin{array}{c}\text { Reaction } \\
\text { Duration, } s\end{array}$ & $\begin{array}{l}\text { Sultur } \\
\text { Absorption, w\% }\end{array}$ & $\begin{array}{l}\text { Reaction } \\
\text { Temperature, }{ }^{\circ} \mathrm{C}\end{array}$ & $\begin{array}{l}\text { Hydrogen } \\
\text { Sulfide, ppm }\end{array}$ & $\begin{array}{l}\text { Hydrogen } \\
\text { v\% }\end{array}$ & $\begin{array}{c}\text { Moisture } \\
\text { v\% }\end{array}$ & $\begin{array}{l}\text { Space } \\
\text { Time, } s\end{array}$ & $\begin{array}{l}\text { Reaction Rate } \\
\text { Constant, om/s }\end{array}$ & $\begin{array}{l}\text { Intraparticle } \\
\text { Diffusivity, cm } / s\end{array}$ \\
\hline 1115 & 0.0102 & 12.51 & 14.44 & & & & & & & \\
\hline 1116 & 0.0103 & 16.2 & 15.57 & & & & & & & \\
\hline 1117 & 0.0104 & 20.33 & 17.8 & & & & & & & \\
\hline 1118 & 0.0108 & 25.42 & 20.46 & & & & & & & \\
\hline 1119 & 0.0107 & 30.23 & 20.92 & & & & & & & \\
\hline 1120 & 0.0104 & 60.34 & 26.83 & & & & & & & \\
\hline 1121 & 0.0105 & 180.34 & 29.34 & & & & & & & \\
\hline 1123 & 0.0056 & 4.36 & 3.886 & 450 & 18000 & 10 & 10 & 0.076 & 1.1134 & 0.001787 \\
\hline 1124 & 0.0054 & 8.4 & 10.07 & & & & & & & \\
\hline 1125 & 0.0053 & 12.46 & 14.34 & & & & & & & \\
\hline 1126 & 0.0052 & 16.17 & 14.52 & & & & & & & \\
\hline 1127 & 0.0057 & 20.2 & 22.63 & & & & & & & \\
\hline 1128 & 0.0053 & 25.33 & 20.61 & & & & & & & \\
\hline 1129 & 0.0054 & 30.18 & 21.36 & & & & & & & \\
\hline 1130 & 0.0056 & 60.2 & 27.44 & & & & & & & \\
\hline 1131 & 0.0056 & 180.47 & 29.56 & & & & & & & \\
\hline 1132 & 0.0154 & 4.34 & 2.239 & 450 & 18000 & 10 & 10 & 0.076 & 0.8855 & 0.002505 \\
\hline 1133 & 0.0153 & 8.6 & 7.733 & & & & & & & \\
\hline 1134 & 0.0155 & 12.2 & 10.47 & & & & & & & \\
\hline 1135 & 0.0152 & 16.28 & 13.71 & & & & & & & \\
\hline 1136 & 0.0151 & 20.14 & 15.79 & & & & & & & \\
\hline 1137 & 0.0152 & 25.24 & 19.41 & & & & & & & \\
\hline 1138 & 0.0154 & 30.48 & 20.41 & & & & & & & \\
\hline 1139 & 0.0156 & 60.2 & 25.84 & & & & & & & \\
\hline 1140 & 0.015 & 180.27 & 28.74 & & & & & & & \\
\hline 1141 & 0.0101 & 4.17 & 2.242 & 450 & 15000 & 10 & 10 & 0.076 & 0.9436 & 0.004041 \\
\hline 1142 & 0.0108 & 8.27 & 5.645 & & & & & & & \\
\hline 1143 & 0.0107 & 12.19 & 10.9 & & & & & & & \\
\hline 1144 & 0.0103 & 16.15 & 13.18 & & & & & & & \\
\hline 1145 & 0.0102 & 20.34 & 16 & & & & & & & \\
\hline 1146 & 0.0106 & 25.12 & 16.9 & & & & & & & \\
\hline 1147 & 0.0104 & 30.22 & 20.19 & & & & & & & \\
\hline 1148 & 0.0104 & 60.14 & 25.79 & & & & & & & \\
\hline 1149 & 0.0108 & 180.26 & 31.68 & & & & & & & \\
\hline 1150 & 0.0103 & 4.39 & 2.017 & 450 & 9000 & 10 & 10 & 0.076 & 1.3843 & 0.003022 \\
\hline 1151 & 0.0103 & 8.31 & 3.372 & & & & & & & \\
\hline 1152 & 0.0101 & 12.41 & 5.578 & & & & & & & \\
\hline 1153 & 0.0105 & 16.38 & 7.639 & & & & & & & \\
\hline 1154 & 0.0107 & 20.14 & 10.84 & & & & & & & \\
\hline 1155 & 0.0108 & 25.37 & 12.19 & & & & & & & \\
\hline 1156 & 0.0102 & 30.14 & 13.78 & & & & & & & \\
\hline 1157 & 0.0109 & 60.34 & 23.42 & & & & & & & \\
\hline 1158 & 0.0104 & 180.24 & 29.17 & & & & & & & \\
\hline 1159 & 0.02 & 4.51 & 2.29 & 450 & 18000 & 10 & 10 & 0.076 & 0.8583 & 0.001924 \\
\hline 1160 & 0.0208 & 8.2 & 5.153 & & & & & & & \\
\hline
\end{tabular}


Table 3. continued-2

\begin{tabular}{|c|c|c|c|c|c|c|c|c|c|c|}
\hline Run \# & $\begin{array}{l}\text { Sorbent } \\
\text { Amount, g }\end{array}$ & $\begin{array}{l}\text { Reaction } \\
\text { Duration, } s\end{array}$ & $\begin{array}{c}\text { Sulfur } \\
\text { Absorption, w\% }\end{array}$ & $\begin{array}{c}\text { Reaction } \\
\text { Temperature, }{ }^{\circ} \mathrm{C}\end{array}$ & $\begin{array}{c}\text { Hydrogen } \\
\text { Sulfide, ppm }\end{array}$ & $\begin{array}{l}\text { Hydrogen } \\
\text { v\% }\end{array}$ & $\begin{array}{c}\text { Moisture } \\
\text { v\% }\end{array}$ & $\begin{array}{l}\text { Space } \\
\text { Time, s }\end{array}$ & $\begin{array}{l}\text { Reaction Rate } \\
\text { Constant, } \mathrm{cm} / \mathrm{s}\end{array}$ & $\begin{array}{l}\text { Intraparticle } \\
\text { Diffusivity, } \mathrm{cm}^{2} / \mathrm{s}\end{array}$ \\
\hline 1161 & 0.0207 & 12.24 & 9.176 & & & & & & & \\
\hline 1162 & 0.0204 & 16.35 & 13.57 & & & & & & & \\
\hline 1163 & 0.0206 & 20.29 & 14.37 & & & & & & & \\
\hline 1164 & 0.0202 & 25.33 & 17.69 & & & & & & & \\
\hline 1165 & 0.0205 & 29.87 & 19.61 & & & & & & & \\
\hline 1166 & 0.0204 & 60.18 & 24.85 & & & & & & & \\
\hline 1167 & 0.0201 & 180.47 & 26.74 & & & & & & & \\
\hline 1168 & 0.0106 & 4.24 & 1.861 & 450 & 18000 & 10 & 20 & 0.076 & 0.9575 & 0.002519 \\
\hline 1169 & 0.0105 & 8.16 & 5.797 & & & & & & & \\
\hline 1170 & 0.0108 & 12.34 & 7.959 & & & & & & & \\
\hline 1171 & 0.0107 & 16.4 & 10.62 & & & & & & & \\
\hline 1172 & 0.0106 & 20.1 & 14.69 & & & & & & & \\
\hline 1173 & 0.0107 & 31.46 & 20.87 & & & & & & & \\
\hline 1174 & 0.0102 & 25.21 & 17.31 & & & & & & & \\
\hline 1175 & 0.0102 & 60.14 & 26.69 & & & & & & & \\
\hline 1176 & 0.0105 & 180.31 & 30.53 & & & & & & & \\
\hline 1177 & 0.0103 & 4.3 & 4.379 & 450 & 18000 & 10 & 5 & 0.076 & 0.8174 & 0.001944 \\
\hline 1178 & 0.0102 & 8.19 & 8.8 & & & & & & & \\
\hline 1179 & 0.01 & 12.25 & 13.07 & & & & & & & \\
\hline 1180 & 0.0104 & 16.16 & 15.95 & & & & & & & \\
\hline 1181 & 0.0105 & 20.35 & 17.13 & & & & & & & \\
\hline 1182 & 0.0107 & 25.06 & 20.71 & & & & & & & \\
\hline 1183 & 0.0105 & 29.99 & 19.8 & & & & & & & \\
\hline 1184 & 0.0105 & 60.14 & 25.41 & & & & & & & \\
\hline 1185 & 0.0105 & 180.07 & 29.07 & & & & & & & \\
\hline 1186 & 0.0103 & 4.21 & 1.907 & 450 & 18000 & 10 & 15 & 0.076 & 0.9071 & 0.002137 \\
\hline 1187 & 0.0102 & 8.38 & 7.569 & & & & & & & \\
\hline 1188 & 0.01 & 12.07 & 10.17 & & & & & & & \\
\hline 1189 & 0.0104 & 16.08 & 12.13 & & & & & & & \\
\hline 1190 & 0.0105 & 20.14 & 16.73 & & & & & & & \\
\hline 1191 & 0.0107 & 25.16 & 17.07 & & & & & & & \\
\hline 1192 & 0.0105 & 30.24 & 20.22 & & & & & & & \\
\hline 1193 & 0.0105 & 60.14 & 26.07 & & & & & & & \\
\hline 1194 & 0.0105 & 180.3 & 28.86 & & & & & & & \\
\hline 1195 & 0.0107 & 4.24 & 2.421 & 450 & 15000 & 10 & 10 & 0.076 & 0.9933 & 0.002510 \\
\hline 1196 & 0.0103 & 8.2 & 4.817 & & & & & & & \\
\hline 1197 & 0.0106 & 12.17 & 10.86 & & & & & & & \\
\hline 1198 & 0.0105 & 16.21 & 13.7 & & & & & & & \\
\hline 1199 & 0.0102 & 20.35 & 13.82 & & & & & & & \\
\hline 1200 & 0.0107 & 25.24 & 17.65 & & & & & & & \\
\hline 1201 & 0.0103 & 30.17 & 19.24 & & & & & & & \\
\hline 1202 & 0.0104 & 60.27 & 25.16 & & & & & & & \\
\hline 1203 & 0.0101 & 180.02 & 29.13 & & & & & & & \\
\hline 1204 & 0.0103 & 4.09 & 1.228 & 375 & 12000 & 10 & 10 & 0.085 & 0.2453 & 0.000622 \\
\hline 1205 & 0.0104 & 8.12 & 1.869 & & & & & & & \\
\hline
\end{tabular}


Table 3. continued-3

\begin{tabular}{|c|c|c|c|c|c|c|c|c|c|c|}
\hline Run \# & $\begin{array}{l}\text { Sorbent } \\
\text { Amount, } g\end{array}$ & $\begin{array}{l}\text { Reaction } \\
\text { Duration, } s\end{array}$ & $\begin{array}{l}\text { Sulfur } \\
\text { Absorption, w\% }\end{array}$ & $\begin{array}{l}\text { Reaction } \\
\text { Temperature, }{ }^{\circ} \mathrm{C}\end{array}$ & $\begin{array}{l}\text { Hydrogen } \\
\text { Sulfide, ppm }\end{array}$ & $\begin{array}{l}\text { Hydrogen } \\
\text { v\% }\end{array}$ & $\begin{array}{c}\text { Moisture } \\
\text { v\% }\end{array}$ & $\begin{array}{c}\text { Space } \\
\text { Time, } s\end{array}$ & $\begin{array}{l}\text { Reaction Rate } \\
\text { Constant, } \mathrm{cm} / \mathrm{s}\end{array}$ & $\begin{array}{l}\text { Intraparticle } \\
\text { Diffusivity, } \mathrm{cm}^{2} / \mathrm{s}\end{array}$ \\
\hline 1206 & 0.0102 & 12.13 & 3.083 & & & & & & & \\
\hline 1207 & 0.0102 & 16.42 & 3.451 & & & & & & & \\
\hline 1208 & 0.0101 & 20.1 & 4.17 & & & & & & & \\
\hline 1209 & 0.0106 & 25.02 & 6.56 & & & & & & & \\
\hline 1210 & 0.0102 & 30.07 & 5.491 & & & & & & & \\
\hline 1211 & 0.0104 & 60.31 & 10.11 & & & & & & & \\
\hline 1212 & 0.0105 & 180.39 & 21.1 & & & & & & & \\
\hline 1213 & 0.0103 & 4.17 & 2.196 & 475 & 12000 & 10 & 10 & 0.073 & 1.1354 & 0.002899 \\
\hline 1214 & 0.0106 & 8.14 & 4.557 & & & & & & & \\
\hline 1215 & 0.01 & 12.05 & 8.316 & & & & & & & \\
\hline 1216 & 0.0101 & 16.11 & 11.53 & & & & & & & \\
\hline 1217 & 0.0102 & 20.15 & 13.09 & & & & & & & \\
\hline 1218 & 0.0101 & 25.17 & 16.56 & & & & & & & \\
\hline 1219 & 0.0101 & 30 & 18.19 & & & & & & & \\
\hline 1220 & 0.0103 & 59.93 & 23.42 & & & & & & & \\
\hline 1221 & 0.0107 & 180.18 & 28.95 & & & & & & & \\
\hline 1222 & 0.0103 & 4.23 & 1.245 & 425 & 12000 & 10 & 10 & 0.079 & 1.0484 & 0.002614 \\
\hline 1223 & 0.01 & 8.27 & 3.604 & & & & & & & \\
\hline 1224 & 0.0102 & 12.1 & 6.156 & & & & & & & \\
\hline 1225 & 0.0106 & 16.06 & 9.076 & & & & & & & \\
\hline 1226 & 0.0102 & 20.21 & 11.51 & & & & & & & \\
\hline 1227 & 0.0104 & 25.21 & 14.85 & & & & & & & \\
\hline 1228 & 0.0107 & 30.2 & 16.78 & & & & & & & \\
\hline 1229 & 0.0109 & 60.28 & 23.18 & & & & & & & \\
\hline 1230 & 0.01 & 180.33 & 28.69 & & & & & & & \\
\hline 1231 & 0.0102 & 4.2 & 1.581 & 400 & 12000 & 10 & 10 & 0.081 & 0.7405 & 0.001863 \\
\hline 1232 & 0.0104 & 8.12 & 2.556 & & & & & & & \\
\hline 1233 & 0.0102 & 12.38 & 5.003 & & & & & & & \\
\hline 1234 & 0.0104 & 16.17 & 6.465 & & & & & & & \\
\hline 1235 & 0.0108 & 20.23 & 5.529 & & & & & & & \\
\hline 1236 & 0.0108 & 25.32 & 10.77 & & & & & & & \\
\hline 1237 & 0.0102 & 25.34 & 8.219 & & & & & & & \\
\hline 1238 & 0.0102 & 30.14 & 13.04 & & & & & & & \\
\hline 1239 & 0.0103 & 60.17 & 20.44 & & & & & & & \\
\hline 1240 & 0.0102 & 180.21 & 28.66 & & & & & & & \\
\hline 1241 & 0.0104 & 4.08 & 1.69 & 450 & 18000 & 20 & 10 & 0.076 & 0.8832 & 0.002365 \\
\hline 1242 & 0.0101 & 8.3 & 5.236 & & & & & & & \\
\hline 1243 & 0.0102 & 12.25 & 12.38 & & & & & & & \\
\hline 1244 & 0.0101 & 16.9 & 15.75 & & & & & & & \\
\hline 1245 & 0.0101 & 20.2 & 17.77 & & & & & & & \\
\hline 1246 & 0.0103 & 25.15 & 15.46 & & & & & & & \\
\hline 1247 & 0.0103 & 30.05 & 21.86 & & & & & & & \\
\hline 1248 & 0.0104 & 60.29 & 26.01 & & & & & & & \\
\hline 1249 & 0.0104 & 180.3 & 29.08 & & & & & & & \\
\hline
\end{tabular}


Table 3. continued-4

\begin{tabular}{|c|c|c|c|c|c|c|c|c|c|c|}
\hline Run \# & $\begin{array}{l}\text { Sorbent } \\
\text { Amount, g }\end{array}$ & $\begin{array}{l}\text { Reaction } \\
\text { Duration, } s\end{array}$ & $\begin{array}{c}\text { Sulfur } \\
\text { Absorption, w\% }\end{array}$ & $\begin{array}{l}\text { Reaction } \\
\text { Temperature, }{ }^{\circ} \mathrm{C}\end{array}$ & $\begin{array}{c}\text { Hydrogen } \\
\text { Sulfide, ppm }\end{array}$ & $\begin{array}{l}\text { Hydrogen } \\
\text { v\% }\end{array}$ & $\begin{array}{l}\text { Moisture } \\
\text { v\% }\end{array}$ & $\begin{array}{l}\text { Space } \\
\text { Time, s }\end{array}$ & $\begin{array}{l}\text { Reaction Rate } \\
\text { Constant, } \mathrm{cm} / \mathrm{s}\end{array}$ & $\begin{array}{l}\text { Intraparticle } \\
\text { Diffusivity, } \mathrm{cm}^{2} / \mathrm{s}\end{array}$ \\
\hline 1250 & 0.0103 & 4.03 & 3.092 & & & & & & & \\
\hline 1251 & 0.0103 & 3.57 & 2.392 & 450 & 18000 & 27 & 10 & 0.076 & 0.8384 & 0.002021 \\
\hline 1252 & 0.0104 & 8.23 & 6.747 & & & & & & & \\
\hline 1253 & 0.0105 & 12.32 & 11.83 & & & & & & & \\
\hline 1254 & 0.0105 & 16.24 & 12.72 & & & & & & & \\
\hline 1255 & 0.0102 & 20.1 & 16.56 & & & & & & & \\
\hline 1256 & 0.01 & 25.33 & 18.98 & & & & & & & \\
\hline 1257 & 0.0106 & 30.12 & 19.93 & & & & & & & \\
\hline 1258 & 0.0102 & 60.16 & 25.16 & & & & & & & \\
\hline 1259 & 0.0106 & 180.17 & 28.68 & & & & & & & \\
\hline 1260 & 0.0103 & 4.31 & 4.729 & 450 & 18000 & 15 & 75 & 0.076 & 0.8802 & 0.001971 \\
\hline 1261 & 0.0103 & 8.14 & 6.653 & & & & & & & \\
\hline 1262 & 0.0101 & 12.25 & 12.4 & & & & & & & \\
\hline 1263 & 0.0106 & 16.13 & 15.39 & & & & & & & \\
\hline 1264 & 0.0106 & 20.15 & 16.66 & & & & & & & \\
\hline 1265 & 0.0103 & 25.3 & 18.87 & & & & & & & \\
\hline 1266 & 0.0106 & 30.3 & 20.88 & & & & & & & \\
\hline 1267 & 0.0105 & 60.3 & 25.98 & & & & & & & \\
\hline 1268 & 0.0106 & 180.37 & 29.04 & & & & & & & \\
\hline 1269 & 0.0106 & 4.19 & 0.842 & 450 & 18000 & 5 & 10 & 0.076 & 1.0054 & 0.002317 \\
\hline 1270 & 0.0108 & 8.18 & 7.318 & & & & & & & \\
\hline 1271 & 0.0104 & 11.92 & 11.69 & & & & & & & \\
\hline 1272 & 0.0101 & 16.16 & 14.7 & & & & & & & \\
\hline 1273 & 0.0106 & 20.3 & 17.86 & & & & & & & \\
\hline 1274 & 0.0104 & 25.17 & 18.46 & & & & & & & \\
\hline 1275 & 0.0108 & 30.34 & 20.5 & & & & & & & \\
\hline 1276 & 0.0106 & 60.35 & 27.47 & & & & & & & \\
\hline 1277 & 0.0106 & 180.14 & 29.31 & & & & & & & \\
\hline 1278 & 0.0101 & 4.28 & 3.028 & & & & & & & \\
\hline
\end{tabular}

Table 4. Surface reaction rate constants and intraparticle diffusivity values calculated with initial absorption data and the reaction model.

\begin{tabular}{cccccccc}
$\begin{array}{c}\text { Temperature, } \\
{ }^{\circ} \mathrm{C}\end{array}$ & $\begin{array}{c}\text { Sorbent } \\
\text { Amount, g }\end{array}$ & $\begin{array}{c}\text { Pressure, } \\
\text { psia }\end{array}$ & \multicolumn{3}{c}{ Concentration, v\% } & Reaction Rate & $\begin{array}{c}\text { Intraparticle } \\
\mathrm{H}_{2} \mathrm{~S}\end{array}$ \\
& & & $\mathrm{H}_{2}$ & $\mathrm{H}_{2} \mathrm{O}$ & Constant cm/s & Diffusivity, cm/s ${ }^{2}$ \\
450 & 0.01 & 40.6 & 1.2 & 10 & 10 & 1.2034 & 0.0029 \\
400 & 0.01 & 40.6 & 1.2 & 10 & 10 & 0.8832 & 0.0017 \\
350 & 0.01 & 40.6 & 1.2 & 10 & 10 & 0.1591 & 0.0002 \\
500 & 0.01 & 40.6 & 1.2 & 10 & 10 & 1.7153 & 0.0020 \\
450 & 0.01 & 40.6 & 1.8 & 10 & 10 & 0.9374 & 0.0023
\end{tabular}


Table 4. Continued-1

\begin{tabular}{|c|c|c|c|c|c|c|c|}
\hline \multirow{2}{*}{$\begin{array}{c}\text { Temperature, } \\
{ }^{\circ} \mathrm{C}\end{array}$} & \multirow{2}{*}{$\begin{array}{c}\text { Sorbent } \\
\text { Amount, } g\end{array}$} & \multirow{2}{*}{$\begin{array}{c}\text { Pressure, } \\
\text { psia }\end{array}$} & \multicolumn{3}{|c|}{ Concentration, v\% } & \multirow{2}{*}{$\begin{array}{l}\text { Reaction Rate } \\
\text { Constant } \mathrm{cm} / \mathrm{s}\end{array}$} & \multirow{2}{*}{$\begin{array}{c}\text { Intraparticle } \\
\text { Diffusivity, } \mathrm{cm} / \mathrm{s}^{2}\end{array}$} \\
\hline & & & $\mathrm{H}_{2} \mathrm{~S}$ & $\mathrm{H}_{2}$ & $\mathrm{H}_{2} \mathrm{O}$ & & \\
\hline 450 & 0.005 & 40.6 & 1.8 & 10 & 10 & 1.1134 & 0.0018 \\
\hline 450 & 0.015 & 40.6 & 1.8 & 10 & 10 & 0.8855 & 0.0025 \\
\hline 450 & 0.01 & 40.6 & 1.5 & 10 & 10 & 0.9436 & 0.0040 \\
\hline 450 & 0.01 & 40.6 & 0.9 & 10 & 10 & 1.3843 & 0.0030 \\
\hline 450 & 0.02 & 40.6 & 1.8 & 10 & 10 & 0.8583 & 0.0019 \\
\hline 450 & 0.01 & 40.6 & 1.8 & 10 & 20 & 0.9575 & 0.0025 \\
\hline 450 & 0.01 & 40.6 & 1.8 & 10 & 5 & 0.8174 & 0.0019 \\
\hline 450 & 0.01 & 40.6 & 1.8 & 10 & 15 & 0.9071 & 0.0021 \\
\hline 450 & 0.01 & 40.6 & 1.5 & 10 & 10 & 0.9933 & 0.0025 \\
\hline 375 & 0.01 & 40.6 & 1.2 & 10 & 10 & 0.2453 & 0.0006 \\
\hline 475 & 0.01 & 40.6 & 1.2 & 10 & 10 & 1.1354 & 0.0029 \\
\hline 425 & 0.01 & 40.6 & 1.2 & 10 & 10 & 1.0484 & 0.0026 \\
\hline 400 & 0.01 & 40.6 & 1.2 & 10 & 10 & 0.7405 & 0.0019 \\
\hline 450 & 0.01 & 40.6 & 1.8 & 20 & 10 & 0.8832 & 0.0024 \\
\hline 450 & 0.01 & 40.6 & 1.8 & 27 & 10 & 0.8384 & 0.0020 \\
\hline 450 & 0.01 & 40.6 & 1.8 & 15 & 10 & 0.8802 & 0.0020 \\
\hline 450 & 0.01 & 40.6 & 1.8 & 5 & 10 & 1.0054 & 0.0023 \\
\hline
\end{tabular}

\section{THEORY}

Levenspiel[1] described a reaction equation for a gas-solid reaction of spherical particles, using three reaction rate-controlling steps such as mass transfer of gaseous reactants through gaseous reaction mixtures, diffusion of reactants through porous solid reactants, and surface reaction of gaseous reactants with solid reactants (see Equation 1). Equation 1 was modified to obtain mass transfer rate of $\mathrm{H}_{2} \mathrm{~S}$, intraparticle diffusivity of $\mathrm{H}_{2} \mathrm{~S}$, and reaction rate of $\mathrm{H}_{2} \mathrm{~S}$ with the sorbent using the physical properties (see Table 2), and the experimental data (see Table 3). This equation is developed under the assumptions that the shrinking core model and a quasisteady state are valid.

$$
\left(-\frac{d\left(\frac{r_{C}}{R}\right)}{d t}\right)^{-1}=\left(\frac{C_{S} R^{2}}{C_{G}}\right)\left(\frac{1}{R k_{g}}-\frac{1}{\delta}\right)\left(\frac{r_{C}}{R}\right)^{2}+\left(\frac{C_{S} R^{2}}{C_{G} \delta}\right)\left(\frac{r_{C}}{R}\right)+\left(\frac{C_{S} R}{C_{G} k_{b}^{\prime}}\right)
$$

Equation 2 is obtained from Equation 1, when mass transfer rates are much higher than both diffusion rates of gaseous reactants and reaction rates of solid reactants. Equation 2 was applied to the experimental data shown in Table 3 to obtain both intraparticle diffusivity of $\mathrm{H}_{2} \mathrm{~S}$ and reaction rate of $\mathrm{H}_{2} \mathrm{~S}$ with the AHI-5 sorbent.

$$
\left(-\frac{d\left(\frac{r_{C}}{R}\right)}{d t}\right)^{-1}=\left(\frac{C_{S} R^{2}}{C_{G} \delta}\right)\left(\frac{r_{C}}{R}-\left(\frac{r_{C}}{R}\right)^{2}\right)+\left(\frac{C_{S} R}{C_{G} k_{b}^{\prime}}\right)
$$


Equations 3 and 6 are obtained from Equation 1, when both intraparticle diffusion controlling step and mass transfer controlling step of gaseous reactants are negligible.

Equation 7 is obtained from Equation 1, when both surface reaction step and mass transfer controlling step of gaseous reactants are negligible.

$$
\begin{aligned}
& \left(-\frac{d\left(\frac{r_{C}}{R}\right)}{d t}\right)^{-1}=\left(\frac{C_{S} R}{C_{G} k_{b}^{\prime}}\right) \\
& t=\left(\frac{C_{S} R}{C_{G} k_{b}^{\prime}}\right)\left(1-\frac{r_{C}}{R}\right) \\
& \left(\frac{r_{C}}{R}\right)=\left(1-x_{B}\right)^{1 / 3} \\
& t=\left(\frac{C_{S} R}{C_{G} k_{b}^{\prime}}\right)\left(1-\left(1-x_{B}\right)^{1 / 3}\right) \\
& t=\left(\frac{C_{S} R^{2}}{6 C_{G} \delta}\right)\left(1-3\left(1-x_{B}\right)^{2 / 3}+2\left(1-x_{B}\right)\right)
\end{aligned}
$$

where

$$
\begin{array}{ll}
\mathrm{r}_{\mathrm{c}}: & \text { radius of an unreacted core } \\
\mathrm{R}: & \text { radius of spherical sorbent } \\
\mathrm{t}: & \text { reaction time } \\
\mathrm{C}_{\mathrm{G}}: & \text { concentration of gaseous reactant } \\
\mathrm{x}_{\mathrm{B}}: & \text { conversion of solid reactant }
\end{array}
$$

$\mathrm{k}_{\mathrm{g}}$ : mass transfer rate of gaseous reactant $\mathrm{C}_{\mathrm{S}}$ : molar concentration of solid reactant

$\delta$ : intraparticle diffusivity of gaseous reactant $\mathrm{k}_{\mathrm{b}}$ : reaction rate constant

\section{RESULTS AND DISCUSSION}

Experiments on absorption of hydrogen sulfide into the AHI-5 sorbent was carried out for $4-10800$ seconds at $350-500^{\circ} \mathrm{C}$ (see Table 1) to evaluate effects of concentration of $\mathrm{H}_{2} \mathrm{~S}, \mathrm{H}_{2}$ and moisture, temperature, and sorbent amount on initial dynamic absorption of hydrogen sulfide into the sorbent. A typical simulated coal gas mixture consists of 12000-ppm hydrogen sulfide, 10 -vol \% moisture, 10 vol \% hydrogen, and nitrogen as remainder. A volumetric feed rate of a simulated coal gas mixture to the reactor is $750 \mathrm{~cm}^{3} / \mathrm{min}$ at room temperature and atmospheric pressure. The temperature of the reactor is controlled in the furnace oven at $350-500^{\circ} \mathrm{C}$. The pressure of the reactor is maintained at 40.7 psia. The total capacity of the sorbent for absorption of $\mathrm{H}_{2} \mathrm{~S}$ is determined with the experimental data shown in Figure 2. Most reaction of $\mathrm{H}_{2} \mathrm{~S}$ with the sobent appears to occur for the first 3-min reaction duration. The entire reaction experimental data for this report were obtained for the 3-min reaction duration. 
Figure 2. Effects of reaction duration on absorption of 12000-ppm $\mathrm{H}_{2} \mathrm{~S}$ on $0.01-\mathrm{g} \mathrm{AHI}-5$ sorbent with $10-\mathrm{v} \%$ hydrogen and $10-\mathrm{v} \%$ moisture at $450^{\circ} \mathrm{C}$ and the space time $0.076 \mathrm{~s}$.

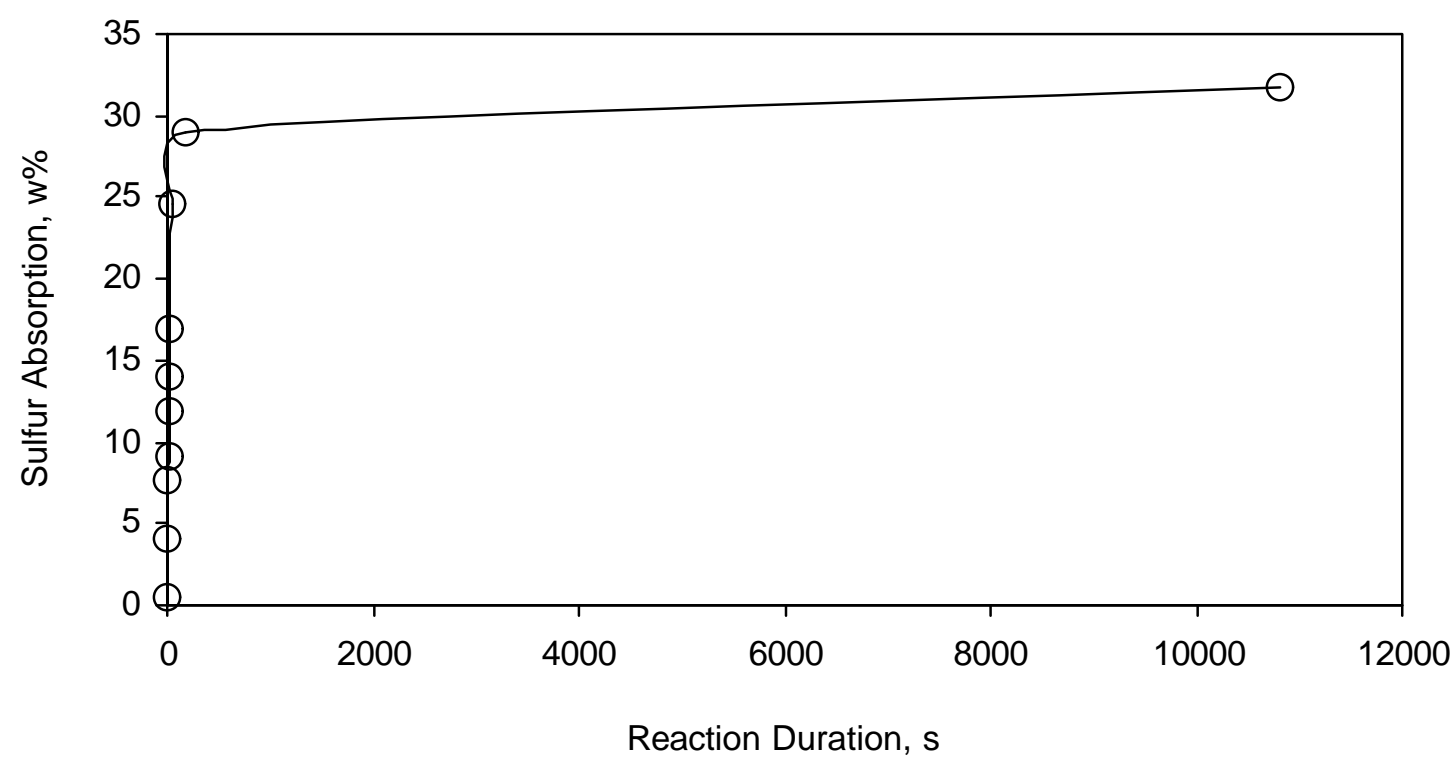

Raw experimental data on absorption of $\mathrm{H}_{2} \mathrm{~S}$ into the sorbent, obtained at various reaction durations and experimental conditions, were shown in Figures 3, 7, 10, 13 and 16.

Conversion of the sorbent, $\mathrm{x}_{\mathrm{B}}$, is calculated by dividing absorption of $\mathrm{H}_{2} \mathrm{~S}$ into the sorbent with the total absorption capacity of the sorbent. The ratio of the radius of an unreacted core $r_{c}$ to the radius of the sorbent $R, r_{c} / R$, is obtained with the conversion value of the sorbent (see Equation 5)

The values of $r_{c} / R$ are plotted against absorption durations, and then the values of ($\left.\mathrm{d}\left(\mathrm{r}_{\mathrm{c}} / \mathrm{R}\right) / \mathrm{dt}\right)^{-1}$ in the left-hand side of Equation 2 are obtained from the values of the slopes of this plotted curve at various reaction durations.

The values of $\left(-d\left(r_{c} / R\right) / d t\right)^{-1}$ as dependent variable and the values of $\left(r_{c} / R-\left(r_{c} / R\right)^{2}\right)$ as independent variable, as shown in Equation 2, are plotted, as shown in Figures 4, 8, 11, 14 and 17 , and then are applied to the linear least squares method to find both the value of the slope and the value of the intercept of this linear curve.

The value of the slope, obtained from the above-mentioned linear least squares method, is equal to the value of $\left(\mathrm{C}_{S} \mathrm{R}^{2} /\left(\mathrm{C}_{\mathrm{G}} \delta\right)\right)$ in Equation 2. The value of intraparticle diffusivity $\delta$ can be computed with the value of the sorbent radius (R), the value of the $\mathrm{H}_{2} \mathrm{~S}$ concentration $\left(\mathrm{C}_{\mathrm{G}}\right)$, and the total absorption capacity of the sorbent $\left(\mathrm{C}_{S}\right)$. The values of intraparticle diffusivity $\delta$ are shown in Table 4. 
The value of the intercept, obtained from the above-mentioned linear least squares method, is equal to the value of $\left(\mathrm{C}_{\mathrm{S}} \mathrm{R} /\left(\mathrm{C}_{\mathrm{G}} \mathrm{k}_{\mathrm{b}}{ }^{\prime}\right)\right)$ in Equation 2. The value of surface reaction rate constant $\mathrm{k}_{\mathrm{b}}$ ' can be computed with the value of the sorbent radius ( $\left.\mathrm{R}\right)$, the value of the $\mathrm{H}_{2} \mathrm{~S}$ concentration $\left(\mathrm{C}_{\mathrm{G}}\right)$, and the total absorption capacity of the sorbent $\left(\mathrm{C}_{\mathrm{S}}\right)$. The values of surface reaction rate constant $\mathrm{k}_{\mathrm{b}}$ ' are shown in Table 4.

Effects of Temperature on Initial Dynamic Absorption of $\mathrm{H}_{2} \mathrm{~S}$

Experiments on initial absorption of $\mathrm{H}_{2} \mathrm{~S}$ into the sorbent were carried out for $4-180$ seconds at 40.7 psia and $350-500^{\circ} \mathrm{C}$ to find effects of temperature on initial dynamic absorption of $\mathrm{H}_{2} \mathrm{~S}$ into the sorbent. The concentrations of both moisture and hydrogen are maintained at 10volume percent. The concentration of hydrogen sulfide in the simulated coal gas mixture is maintained at $12000 \mathrm{ppm}$.

Initial absorption of $\mathrm{H}_{2} \mathrm{~S}$ into the AHI-5 sorbent is affected with absorption temperature for short absorption duration of 4 - 180 seconds (see Figure 3). Equation 2 was applied to the experimental data to obtain surface reaction rate constants of $\mathrm{H}_{2} \mathrm{~S}$ with the sorbent and intraparticle diffusivity values through pores of the sorbent (see Figure 4). The range of surface reaction rate constant is $0.16-1.2 \mathrm{~cm} / \mathrm{s}$ (see Table 4 ). The range of intraparticle diffusivity values of the sorbent is $0.0002-0.0029 \mathrm{~cm}^{2} / \mathrm{s}$. These observations may indicate that reactivity of the AHI-5 sorbent be dependent on reaction temperature in the temperature range of $350-$ $500^{\circ} \mathrm{C}$. However, reactivity of the sorbent is much lower at $350^{\circ} \mathrm{C}$ than that at $500^{\circ} \mathrm{C}$. These facts also suggest that the controlling steps for the initial absorption of $\mathrm{H}_{2} \mathrm{~S}$ into sorbent particles are surface reaction of $\mathrm{H}_{2} \mathrm{~S}$ with reactive sites of sorbent particles and intraparticle diffusivity through pores of the sorbent.

Figure 3. Effects of temperature on absorption of 12000-ppm $\mathrm{H}_{2} \mathrm{~S}$ on $0.01-\mathrm{g}$ AHI-5 sorbent with $10-v \%$ hydrogen and $10-v \%$ moisture.

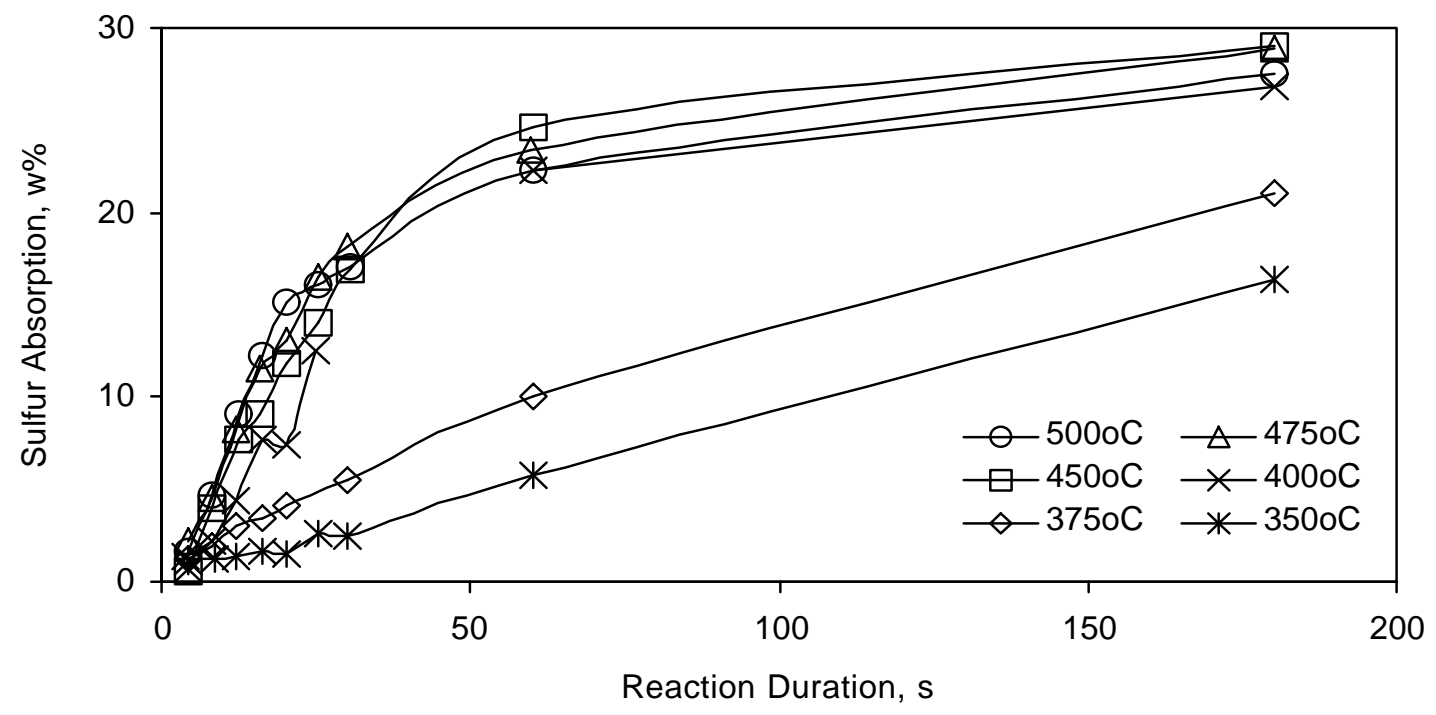


Figure 4. Effects of temperature on absorption of 12000-ppm $\mathrm{H}_{2} \mathrm{~S}$ on $0.01-\mathrm{g}$ AHI-5 sorbent with $10-\mathrm{v} \%$ hydrogen and $10-\mathrm{v} \%$ moisture, using the reaction model.

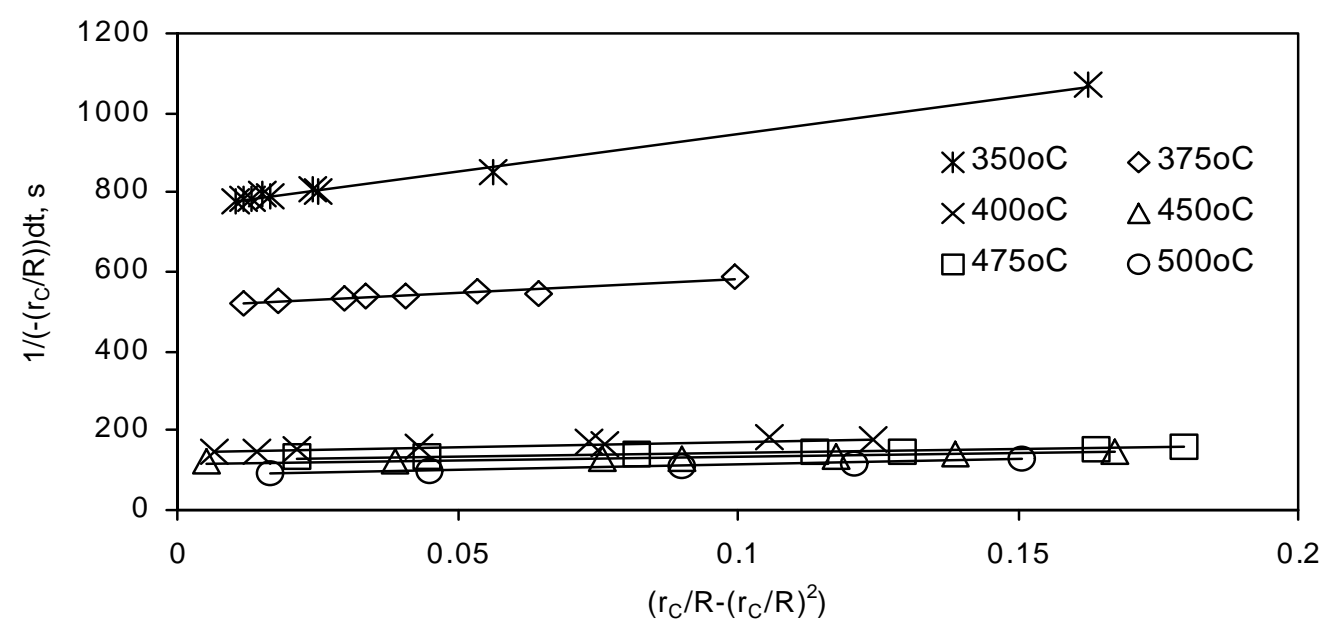

The surface reaction rate constant and the intraparticle diffusivity value increases with the reaction temperatures up to $450^{\circ} \mathrm{C}$. However, the surface reaction rate constant increases steeply, and the intraparticle diffusivity value decreases in the temperature range of $475-500^{\circ} \mathrm{C}$. These facts may indicate that collapsing the pore structure of the sorbent results in the decreased intraparticle diffusivity value in the temperature range of $475-500^{\circ} \mathrm{C}$. The surface reaction rate constant and the intraparticle diffusivity value levels off in the temperature range of $425-475^{\circ} \mathrm{C}$. This observation may indicate that both surface reaction rate constant and intraparticle diffusivity value appear to independent of absorption temperature.

Figure 5. Effects of temperature on surface reaction rate constant and intraparticle diffusivity in the absorption of $12000-\mathrm{ppm}_{2} \mathrm{~S}$ into AHI-5 sorbent with $10-\mathrm{v} \% \mathrm{H}_{2}$ and $10-\mathrm{v} \%$ moisture.

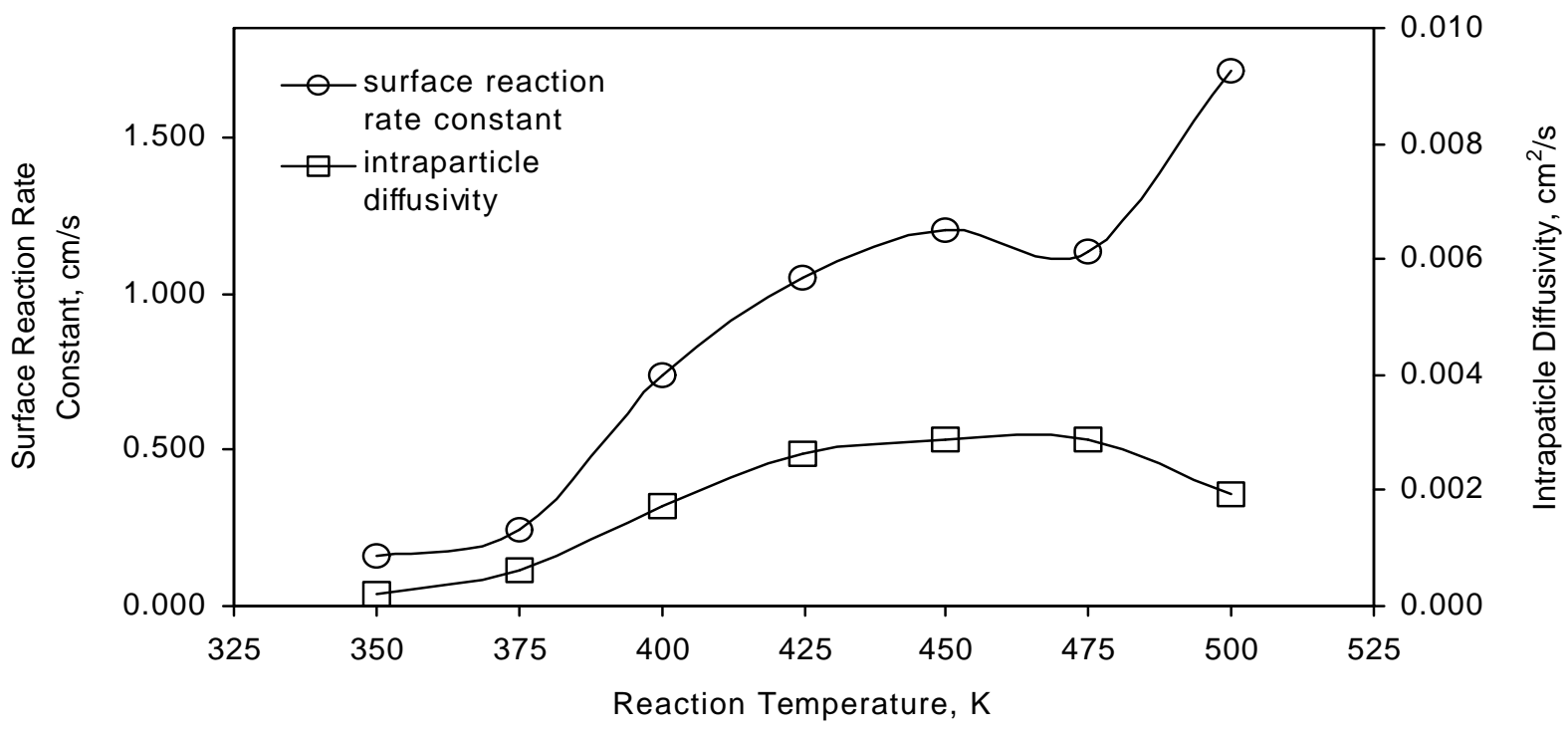


The surface reaction rate constants, obtained from the reaction model, are applied to the Arrhenius equation (see Figure 6). The surface reaction rate constants appear to agree fairly with the equation in the temperature range of $350-475^{\circ} \mathrm{C}$. However, the surface reaction rate constant at $500^{\circ} \mathrm{C}$ is not agreeable with the equation, and is not included in Figure 6.

Figure 6. Effects of temperature on surface reaction rate constant in the absorption of 12000-ppm $\mathrm{H}_{2} \mathrm{~S}$ into AHI-5 sorbent with 10-v\% $\mathrm{H}_{2}$ and 10-v\% moisture, using the Arrhenius equation.

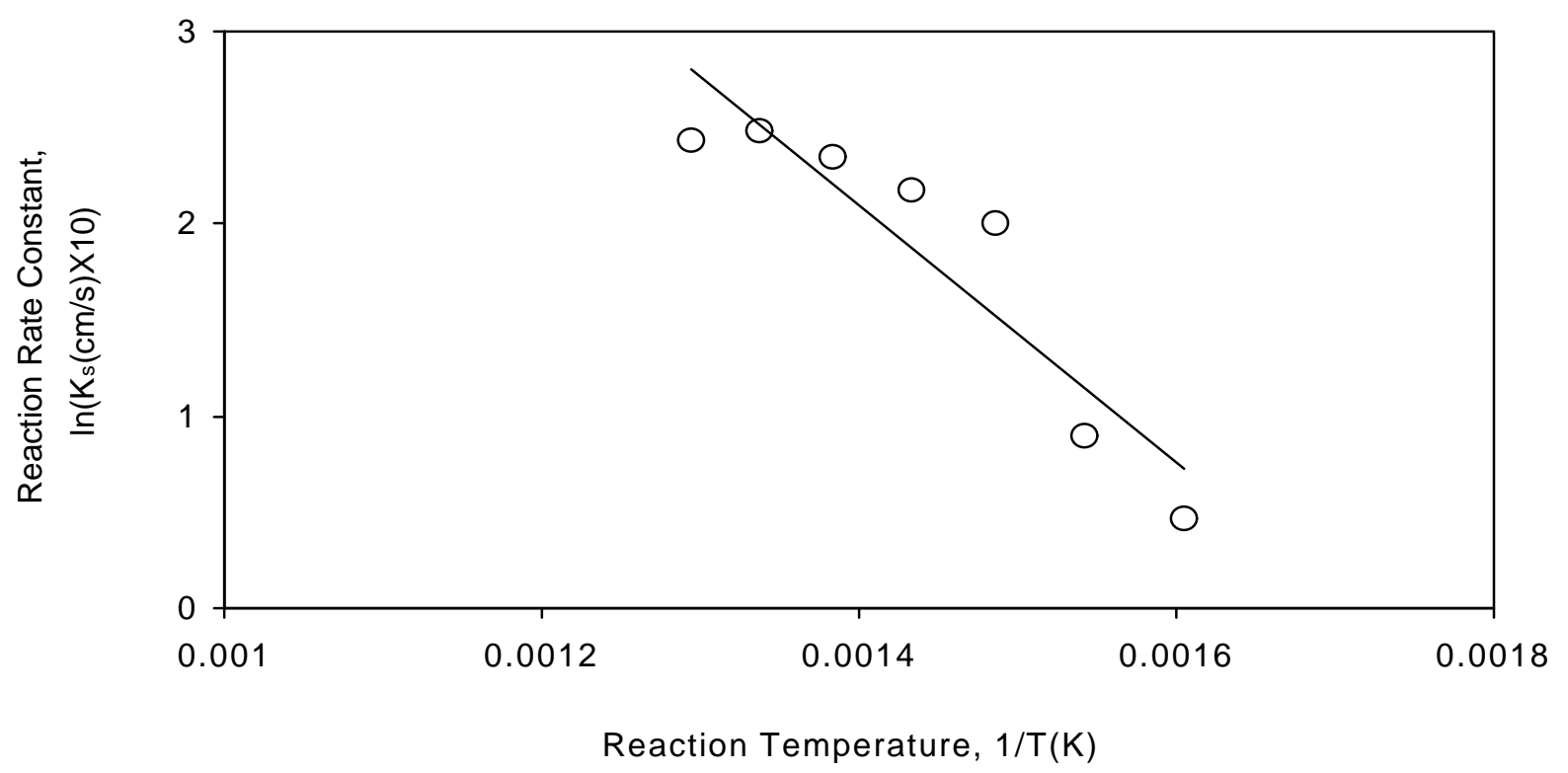

Effects of Sorbent amounts on Initial Dynamic Absorption of $\mathrm{H}_{2} \mathrm{~S}$

Experiments on effects of sorbent amounts on initial dynamic absorption of $\mathrm{H}_{2} \mathrm{~S}$ into the sorbent were conducted for the reaction duration of $4-180$ seconds at $450^{\circ} \mathrm{C}$ and the space-time of $0.076 \mathrm{~s}$. Amounts of the AHI-5 sorbent affect slightly initial dynamic absorption of $\mathrm{H}_{2} \mathrm{~S}$ into the sorbent in the sorbent amount range of 0.005- $0.02 \mathrm{~g}$ (see Figure 7). Equation 2 was applied to the experimental data to obtain surface reaction rate constants and intraparticle diffusivity values of $\mathrm{H}_{2} \mathrm{~S}$ (see Figure 8). The range of the surface reaction rate constant is $0.86-1.11 \mathrm{~cm} / \mathrm{s}$, and the range of the intraparticle diffusivity values is $0.0018-0.0025 \mathrm{~cm}^{2} / \mathrm{s}$. The concentrations of both hydrogen and moisture were maintained at 10 -v percent. The concentration of $\mathrm{H}_{2} \mathrm{~S}$ was maintained at $18000 \mathrm{ppm}$. Absorption of $\mathrm{H}_{2} \mathrm{~S}$ into the sorbent increases with decreased amount of the sorbent in the reactor (see Figure 7). The surface reaction rate constant decrease with increased amount of the sorbent. The intraparticle diffusity values increase generally with increased amount of the sorbent (see Figure 9).

These observations may suggest that mass transfer of $\mathrm{H}_{2} \mathrm{~S}$ from the bulk gaseous phase to the surface of the solid sorbent affects somewhat the overall reaction of $\mathrm{H}_{2} \mathrm{~S}$ with the sorbent 
Figure 7. Effects of sorbent amount on absorption of 18000-ppm $\mathrm{H}_{2} \mathrm{~S}$ on $\mathrm{AH}-$ 5 sorbent with $10-\mathrm{v} \%$ hydrogen and $10-\mathrm{v} \%$ moisture at $450^{\circ} \mathrm{C}$ and the space time $0.076 \mathrm{~s}$.

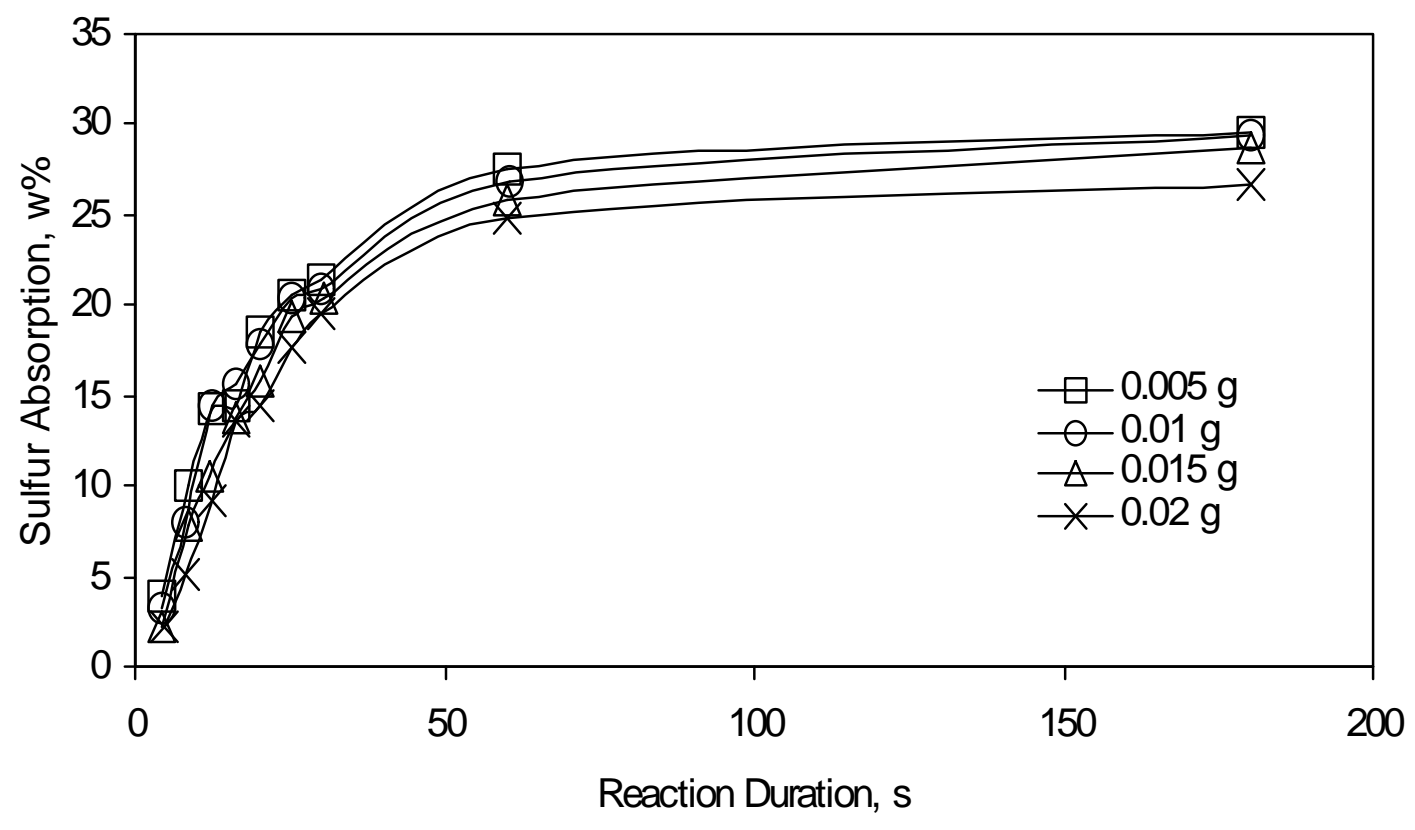

Figure 8. Effects of sorbent amount on absorption of 18000-ppm $\mathrm{HLS}$ on AHI-5 sorbent with 10 -v\% hydrogen and $10-\mathrm{v} \%$ moisture at $450^{\circ} \mathrm{C}$ and the space time $0.076 \mathrm{~s}$, using the reaction model.

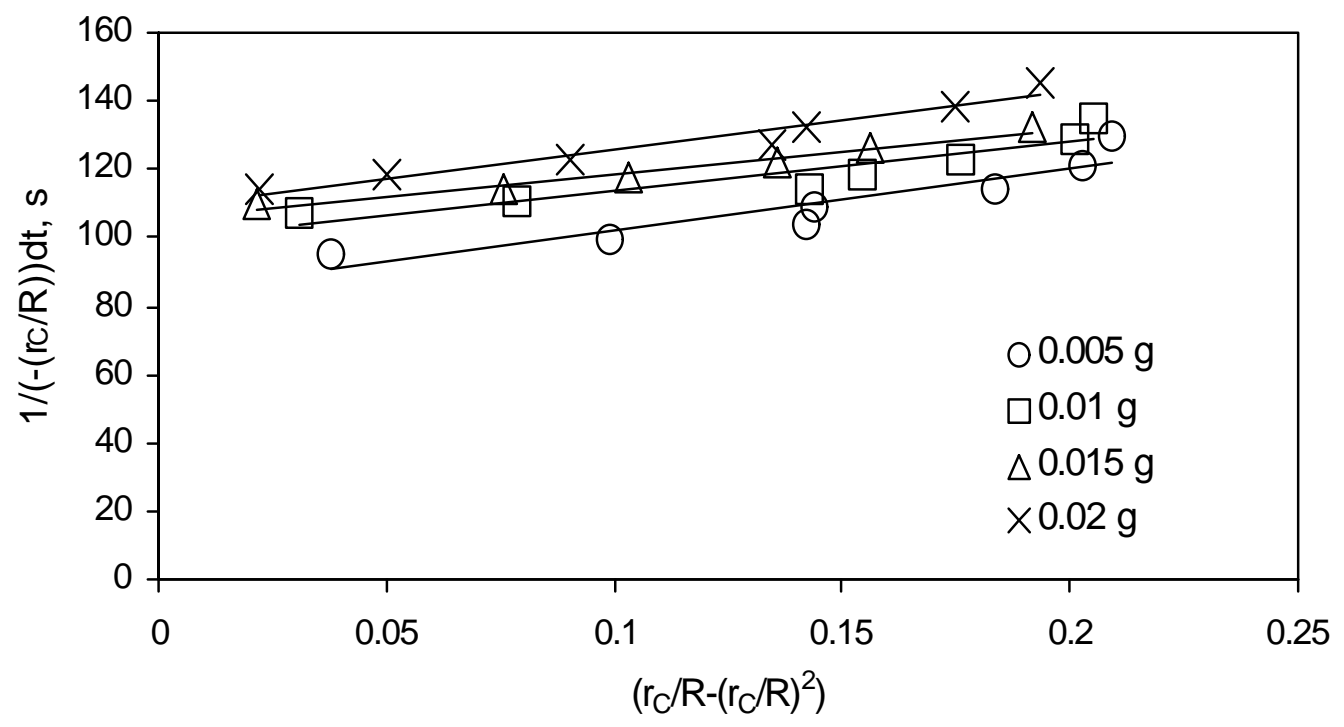


Figure 9. Effects of sorbent amount on surface reaction rate constant and intraparticle diffusivity in the absorption of $18000-\mathrm{ppm} \mathrm{H}_{2} \mathrm{~S}$ into AHI-5 sorbent with 10 v\% $\mathrm{H}_{2}$ and $10-\mathrm{v} \%$ moisture at $450^{\circ} \mathrm{C}$.

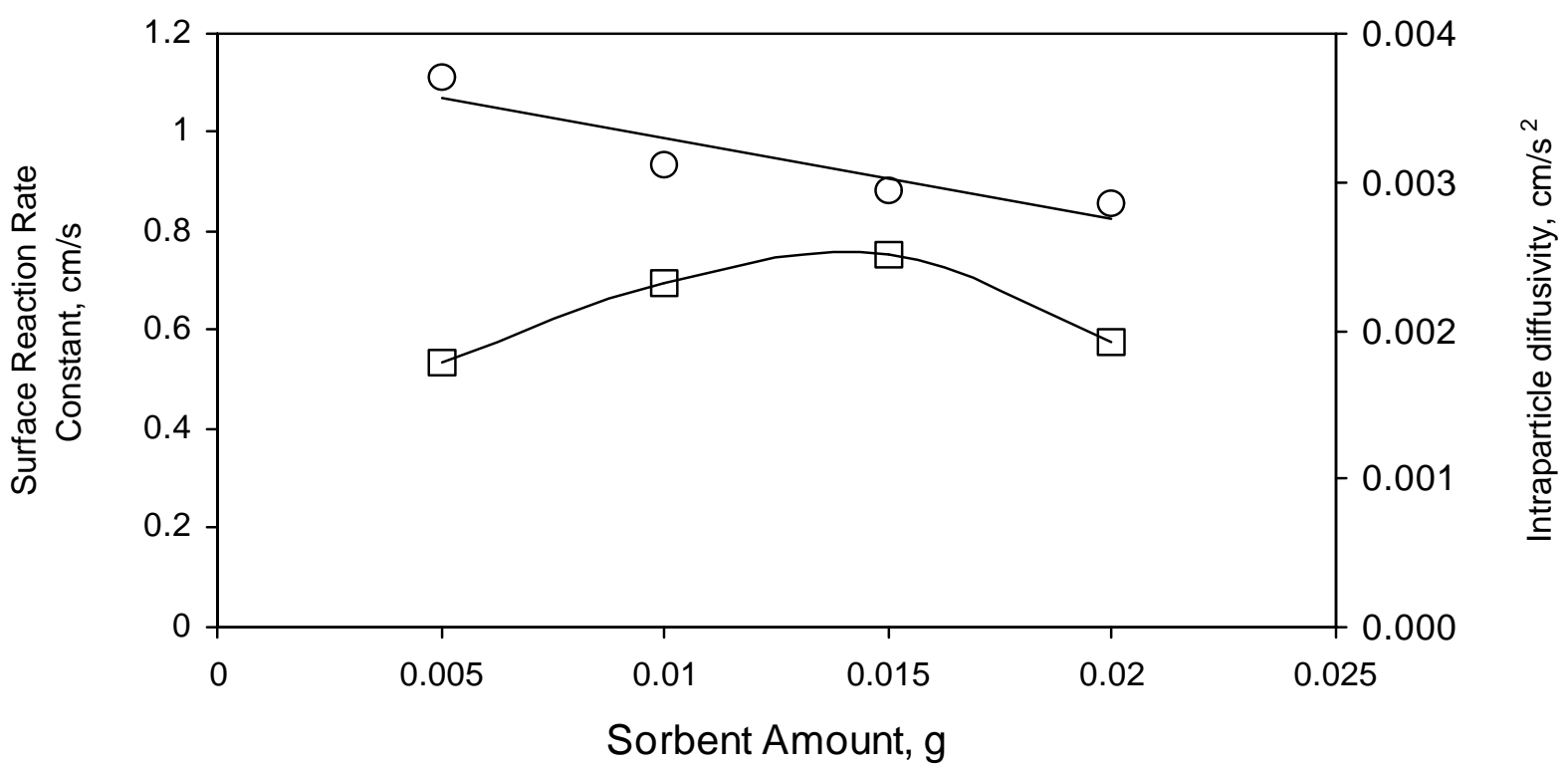

Figure 10. Effects of $\mathrm{H}_{2} \mathrm{~S}$ concentration on absorption of $\mathrm{H}_{2} \mathrm{~S}$ on $0.01-\mathrm{g} \mathrm{AHI-}$ 5 sorbent with $10-v \%$ hydrogen and $10-v \%$ moisture at $450^{\circ} \mathrm{C}$ and the space time $0.076 \mathrm{~s}$.

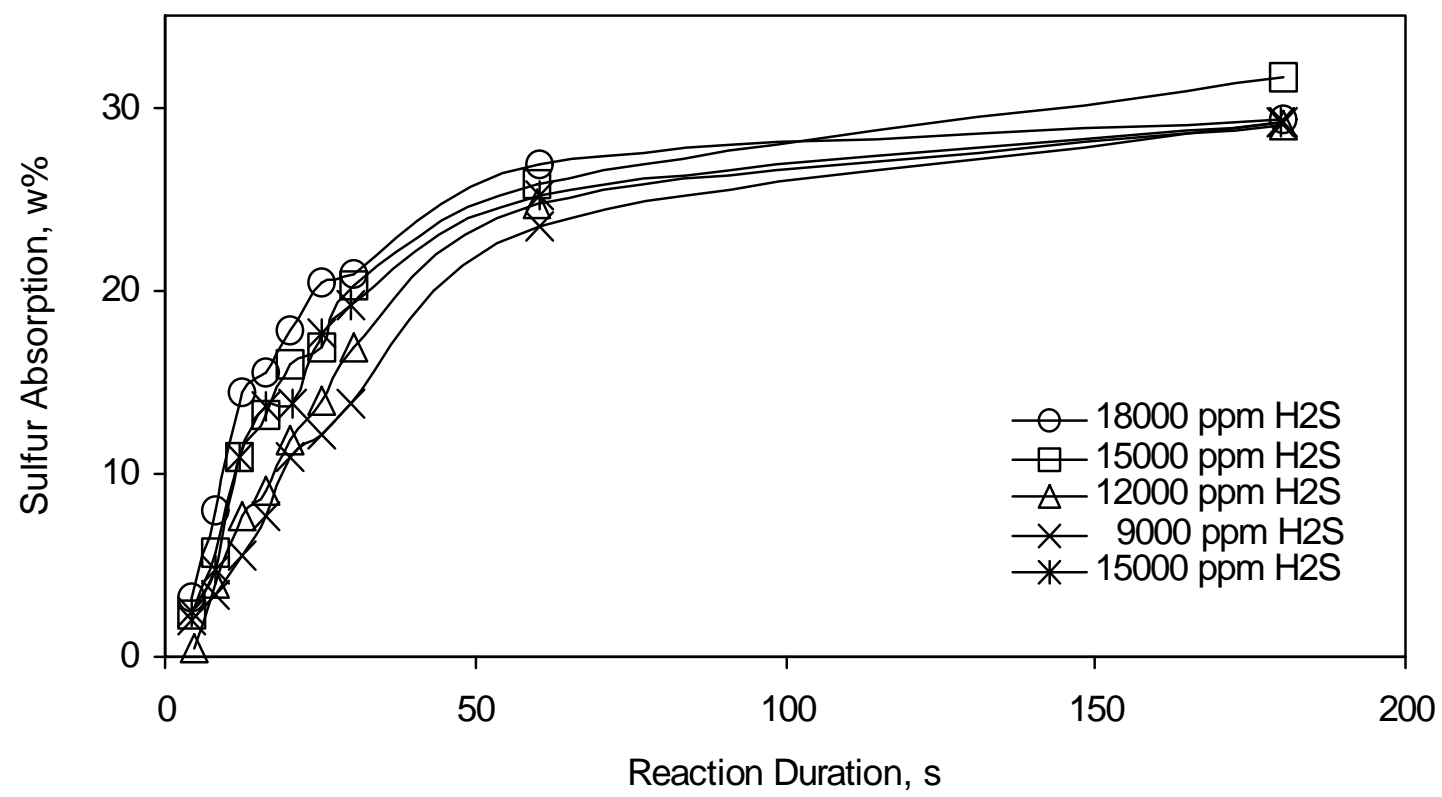




\section{Effects of Concentration of $\mathrm{H}_{2} \mathrm{~S}$ on Initial Dynamic Absorption of $\mathrm{H}_{2} \mathrm{~S}$}

Experiments on effects of concentration of $\mathrm{H}_{2} \mathrm{~S}$ on initial dynamic absorption of $\mathrm{H}_{2} \mathrm{~S}$ into the sorbents were conducted for the reaction duration of $4-180$ seconds at $450^{\circ} \mathrm{C}$ and the spacetime of $0.076 \mathrm{~s}$. The concentrations of hydrogen and moisture were maintained at 10 -volume percent, respectively

Figure 11. Effects of $\mathrm{H}_{2} \mathrm{~S}$ concentration on absorption of $\mathrm{H}_{2} \mathrm{~S}$ on $0.01-\mathrm{g} \mathrm{AHI}$ 5 sorbent with $10-\mathrm{v} \%$ hydrogen and $10-\mathrm{v} \%$ moisture at $450^{\circ} \mathrm{C}$ and the space time $0.076 \mathrm{~s}$, using the reaction model.

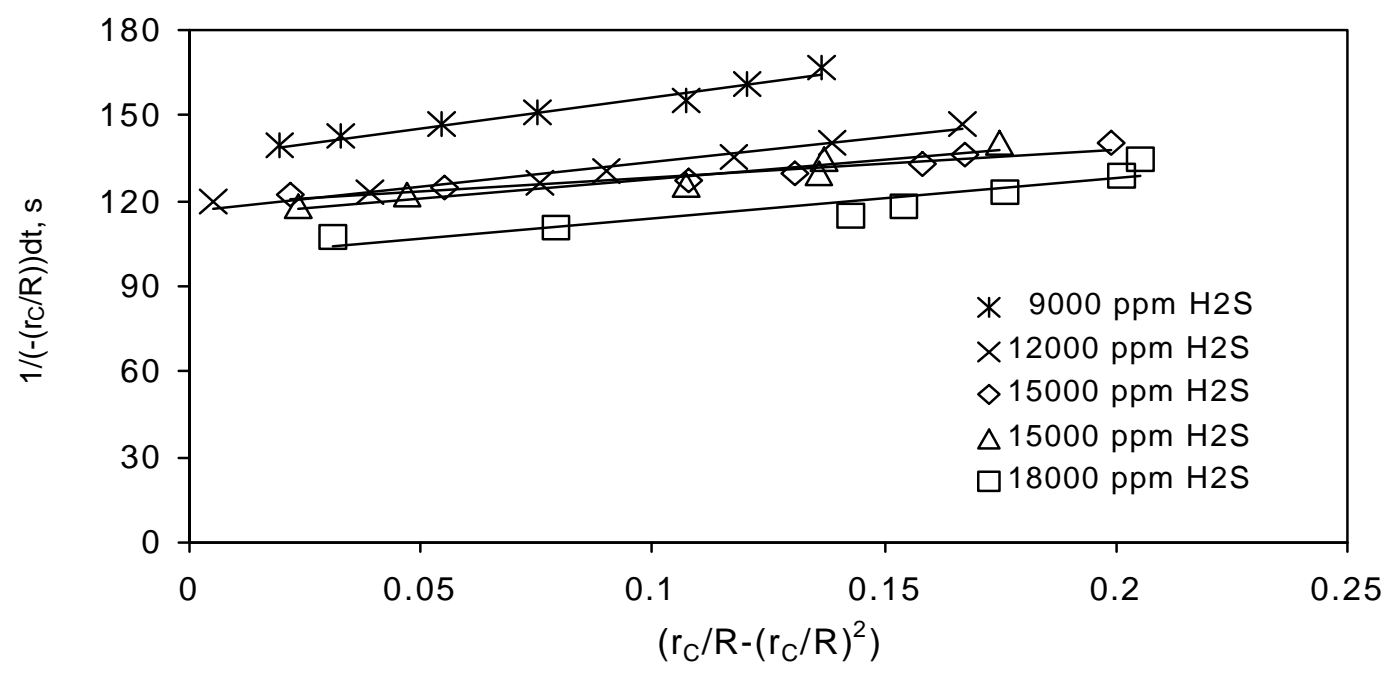

Figure 12. Effects of $\mathrm{H}_{2} \mathrm{~S}$ concentration on surface reaction rate constant and intraparticle diffusivity in the absorption of $\mathrm{H}_{2} \mathrm{~S}$ into 0.01 -g AHI-5 sorbent with $10-\mathrm{v} \%$ $\mathrm{H}_{2}$ and $10-\mathrm{v} \%$ moisture at $450^{\circ} \mathrm{C}$.

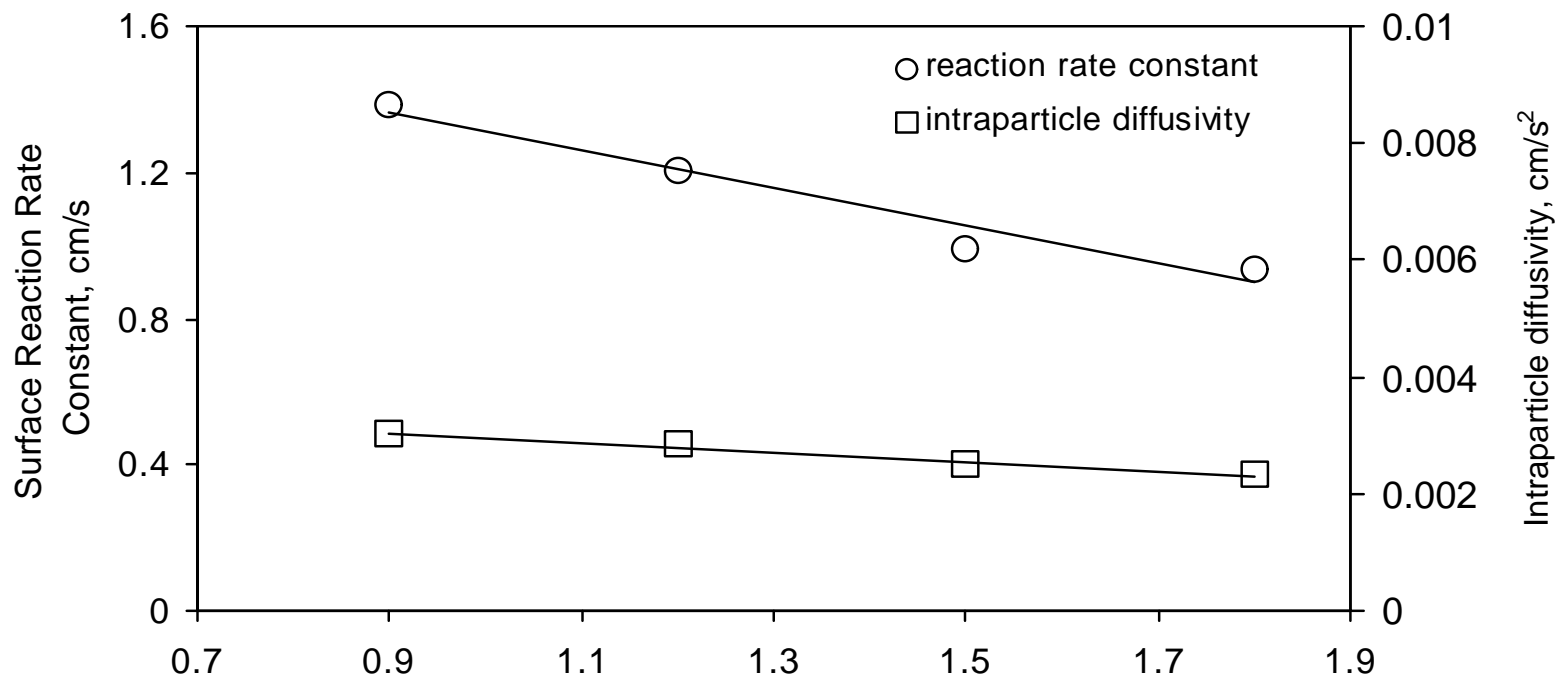

$\mathrm{H}_{2} \mathrm{~S}$ Concentration, \% 
Concentrations of $\mathrm{H}_{2} \mathrm{~S}$ affect significantly initial dynamic absorption of $\mathrm{H}_{2} \mathrm{~S}$ into the AHI-5 sorbent in the $\mathrm{H}_{2} \mathrm{~S}$ concentration range of $9000-18000 \mathrm{ppm}$ (see Figure 10). Equation 2 is applied to the experimental data. The range of surface reaction rate constant is $0.94-1.38$ $\mathrm{cm} / \mathrm{s}$, and the range of intraparticle diffusivity values is $0.0023-0.0031 \mathrm{~cm}^{2} / \mathrm{s}$. The reaction rate constants of the sorbent, obtained from Equations 2, decrease with increased $\mathrm{H}_{2} \mathrm{~S}$ concentration (see Figure 12). The intraparticle diffusivity values of $\mathrm{H}_{2} \mathrm{~S}$ through porous sorbents also decrease with increased $\mathrm{H}_{2} \mathrm{~S}$ concentrations in the $\mathrm{H}_{2} \mathrm{~S}$ concentration range of $9000-18000$ ppm.

Figure 13. Effects of moisture concentration on absorption of 18000 -ppm $\mathrm{H}_{2} \mathrm{~S}$ on $0.01-\mathrm{g} \mathrm{AHI}-5$ sorbent with $10-\mathrm{v} \%$ hydrogen at $450^{\circ} \mathrm{C}$ and the space time $0.076 \mathrm{~s}$.

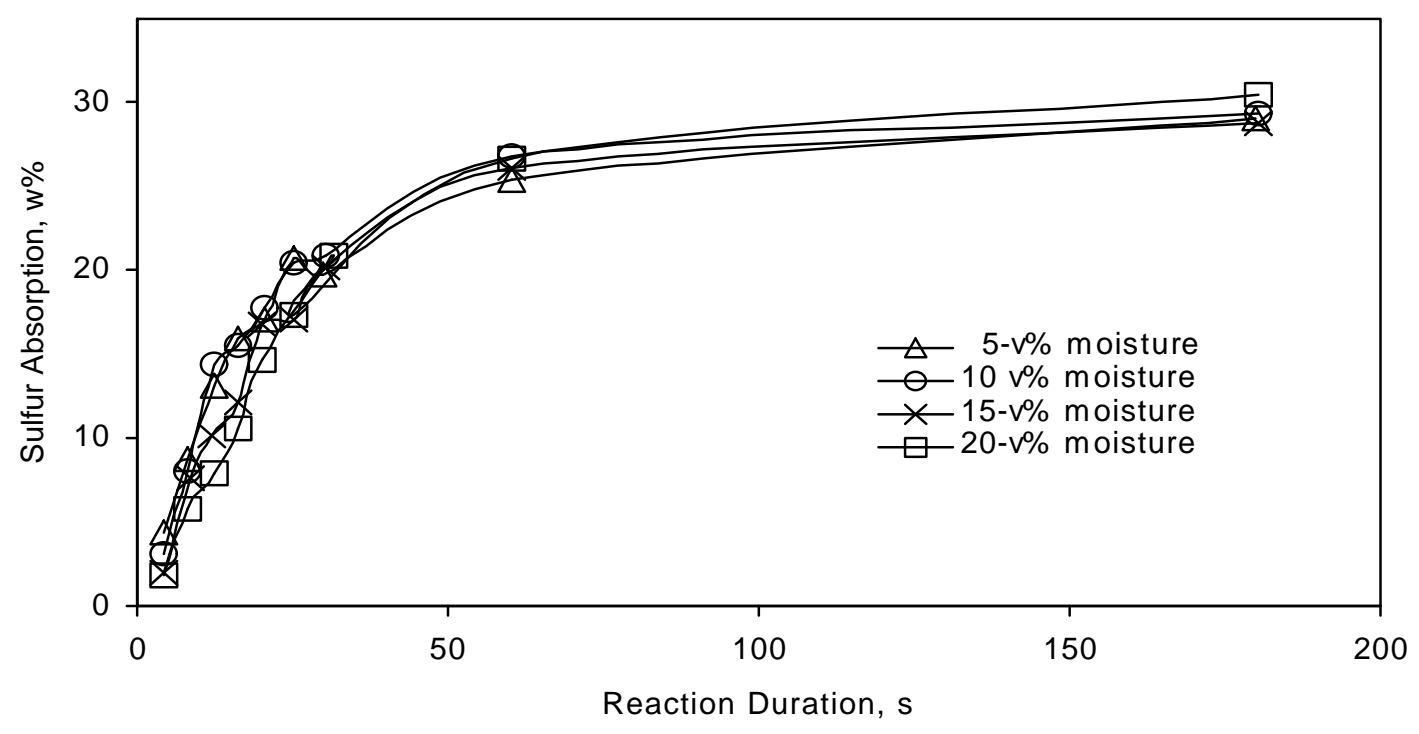

Figure 14. Effects of moisture concentration on absorption of $18000-p p m \mathrm{H}_{2} \mathrm{~S}$ on 0.01 -g AHI-5 sorbent with $10-v \%$ hydrogen at $450^{\circ} \mathrm{C}$ and the space time $0.076 \mathrm{~s}$, using the reaction model.

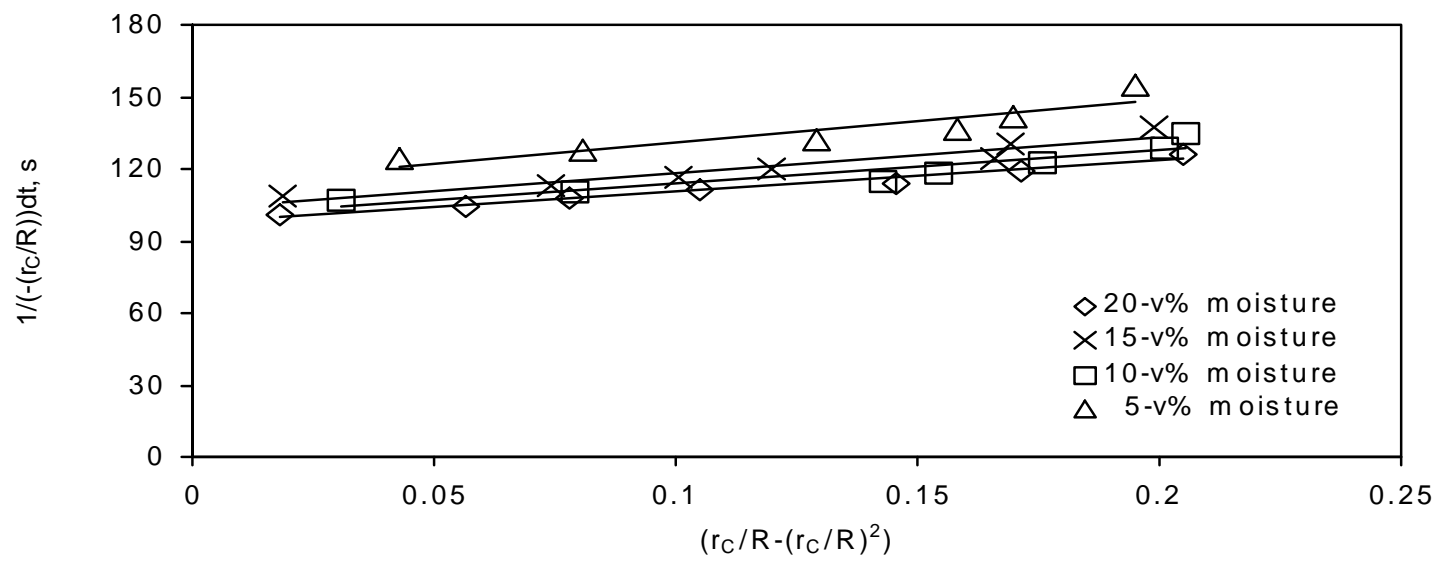


Effects of Moisture on Initial Dynamic Absorption of $\mathrm{H}_{2} \mathrm{~S}$

Experiments on initial absorption of $\mathrm{H}_{2} \mathrm{~S}$ into the sorbents were carried out for $4-180$ seconds at $40.7 \mathrm{psia}$ and $450^{\circ} \mathrm{C}$ to find effects of moisture concentrations on initial dynamic absorption of $\mathrm{H}_{2} \mathrm{~S}$ with the sorbent. The concentrations of $\mathrm{H}_{2} \mathrm{~S}$ and hydrogen are maintained at $18000 \mathrm{ppm}$ and 10-vol \%, respectively. The concentration range of moisture in the simulated coal gas mixture is $5-20$ volume percent.

Figure 15. Effects of moisture concentration on surface reaction rate constant and intraparticle diffusivity in the absorption of $\mathrm{H}_{2} \mathrm{~S}$ into 0.01 -g AHI-5 sorbent with 10 -v\% $\mathrm{H}_{2}$ at $450^{\circ} \mathrm{C}$.

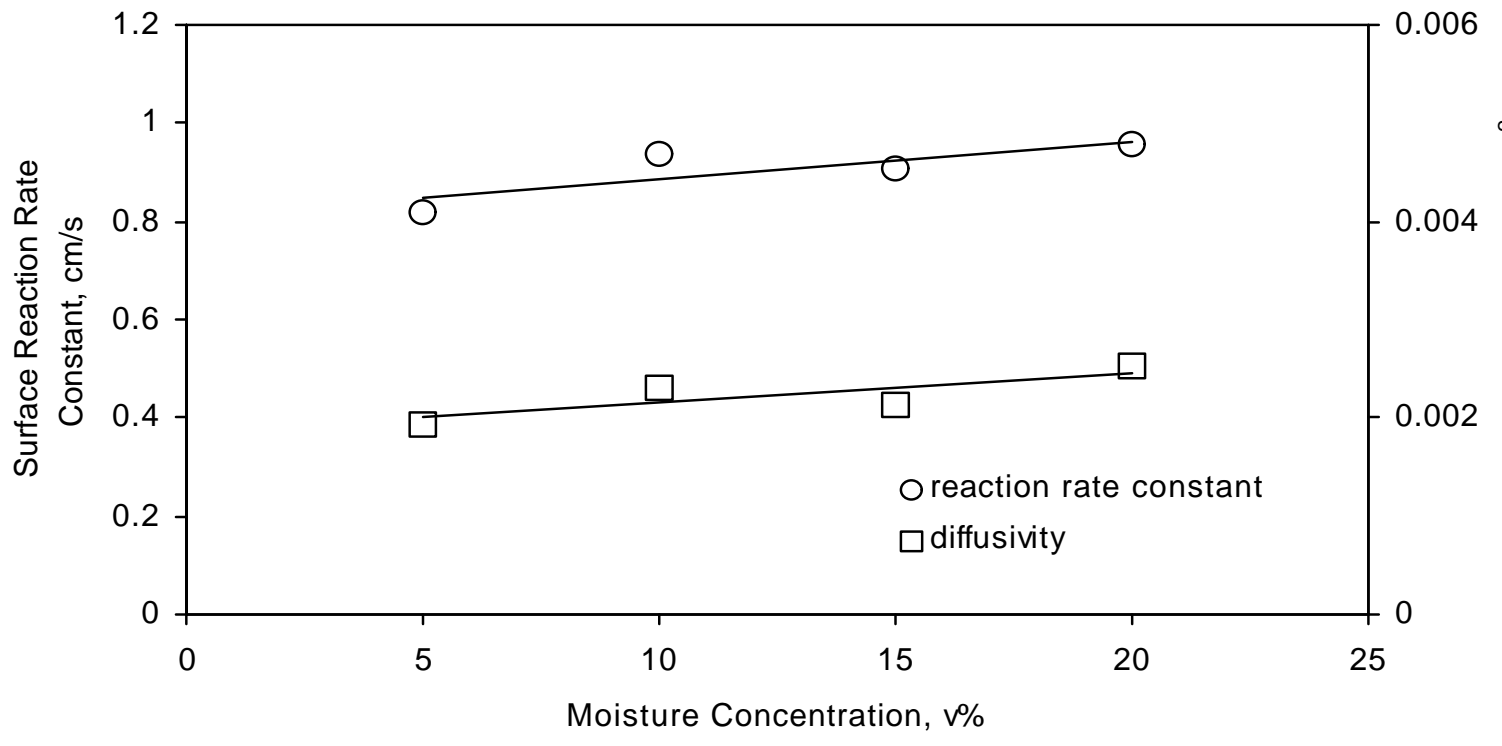

Initial absorption of $\mathrm{H}_{2} \mathrm{~S}$ into the AHI-5 sorbent is affected with concentrations of moisture for short absorption duration of $4-180$ seconds (see Figure 13). These facts may indicate that moisture is not inert for the reaction of $\mathrm{H}_{2} \mathrm{~S}$ with the AHI-5 sorbent. Equation 2 was applied to the experimental data to obtain surface reaction rate constants and intraparticle diffusivity values of $\mathrm{H}_{2} \mathrm{~S}$ with the sorbent (see Figure 14). The range of surface reaction rate constants is $0.82-0.96 \mathrm{~cm} / \mathrm{s}$, and the range of intraparticle diffusivity values is $0.0019-0.0025$ $\mathrm{cm}^{2} / \mathrm{s}$ (see Table 4). These observations may indicate that reactivity of the AHI-5 sorbent and intraparticle diffusivity increase slightly with increased moisture concentrations. These facts also suggest that the controlling steps for the absorption of $\mathrm{H}_{2} \mathrm{~S}$ into sorbent particles are surface reaction of $\mathrm{H}_{2} \mathrm{~S}$ with reactive sites of sorbent particles and diffusion of $\mathrm{H}_{2} \mathrm{~S}$ through porous sorbent particles in the presence of moisture.

\section{Effects of Hydrogen Concentration on Initial Dynamic Absorption of $\mathrm{H}_{2} \mathrm{~S}$}

Experiments on effects of hydrogen concentrations on initial dynamic absorption of $\mathrm{H}_{2} \mathrm{~S}$ into the sorbents were conducted for the reaction duration of $4-180 \mathrm{~s}$ at $450^{\circ} \mathrm{C}$ and the space- 
time of $0.076 \mathrm{~s}$. The concentration of moisture was maintained at 10 -volume percent. The concentration of $\mathrm{H}_{2} \mathrm{~S}$ was maintained at $18000 \mathrm{ppm}$.

Figure 16. Effects of hydrogen concentration on absorption of 18000-ppm $\mathrm{H}_{2} \mathrm{~S}$ on $0.01-\mathrm{g} \mathrm{AHI}-5$ sorbent with $10-\mathrm{v} \%$ moisture at $450^{\circ} \mathrm{C}$ and the space time $0.076 \mathrm{~s}$.

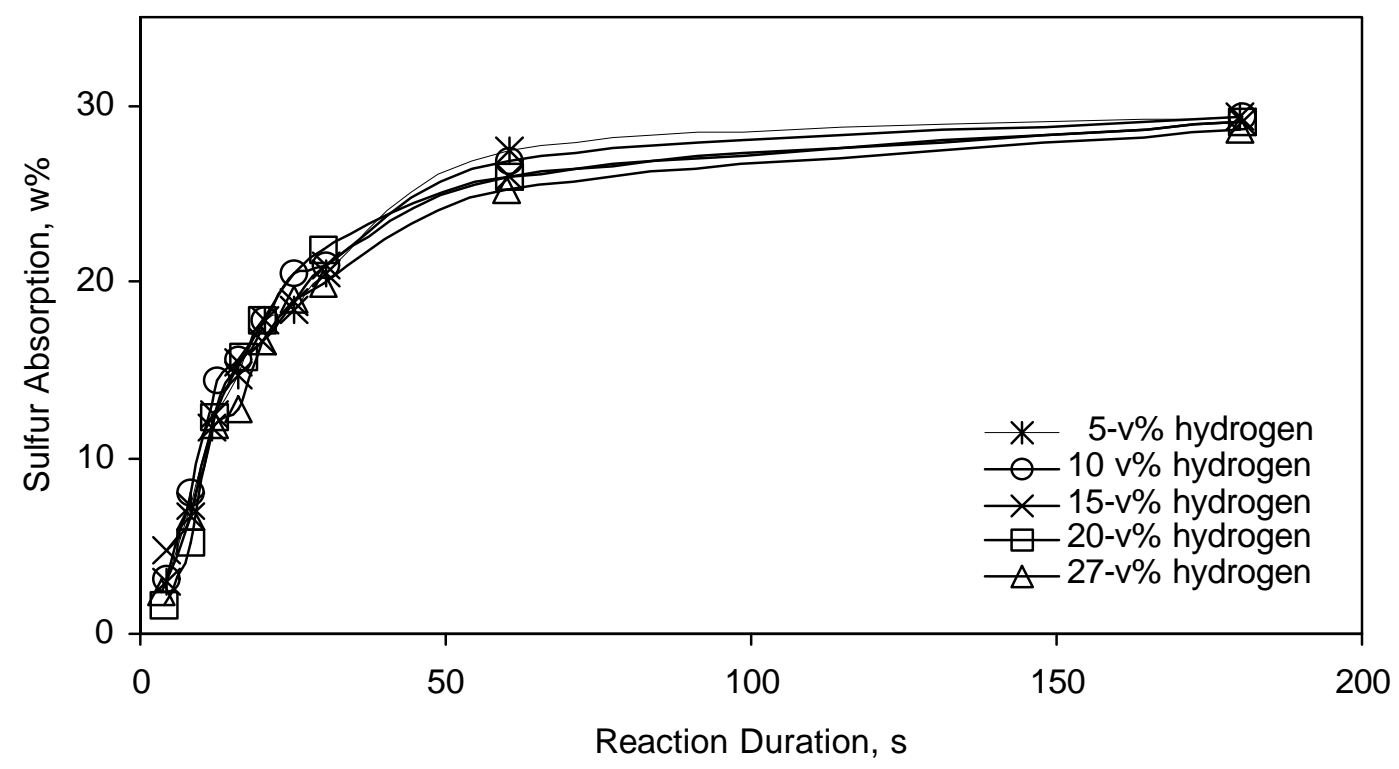

Figure 17. Effects of hydrogen concentration on absorption of 18000-ppm $\mathrm{H}_{2} \mathrm{~S}$ on $0.01-\mathrm{g} \mathrm{AHI}-5$ sorbent with $10-\mathrm{v} \%$ moisture at $450^{\circ} \mathrm{C}$ and the space time $0.076 \mathrm{~s}$, using the reaction model.

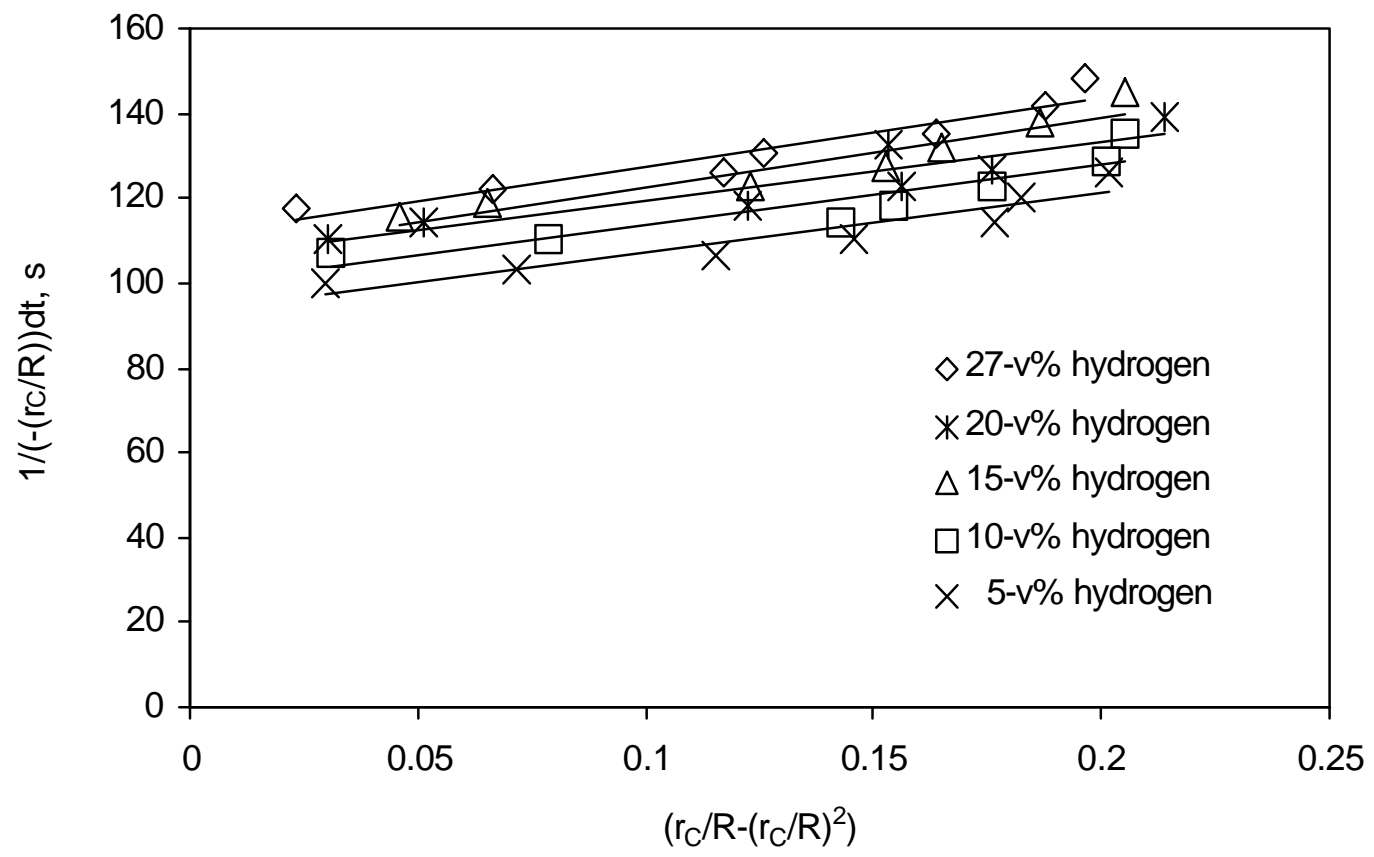


Figure 18. Effects of hydrogen concentration on surface reaction rate constant and intraparticle diffusivity in the absorption of $\mathrm{H}_{2} \mathrm{~S}$ into $0.01-\mathrm{g} \mathrm{AHI}-5$ sorbent with 10 -v\% moisture at $450^{\circ} \mathrm{C}$.

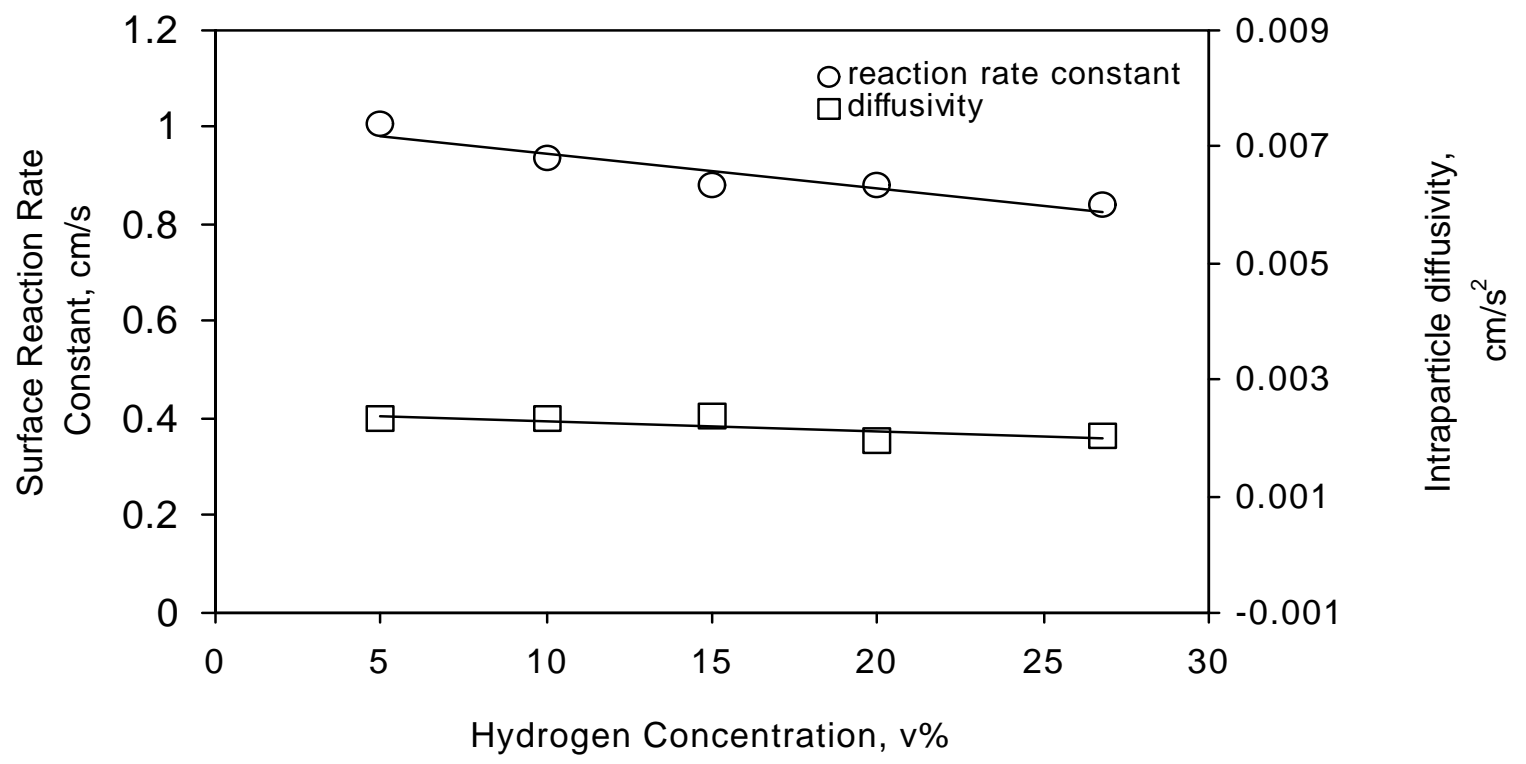

Concentrations of hydrogen affect slightly initial dynamic absorption of $\mathrm{H}_{2} \mathrm{~S}$ into the AHI-5 sorbent in the hydrogen concentration range of 5-27 v\% (see Figure 16). Equation 2 was applied to the experimental data to obtain initial surface reaction rate constants and intraparticle diffusivity values (see Figure 17). The range of the initial surface reaction rate constant is $0.84-1.01 \mathrm{~cm} / \mathrm{s}$, and the range of the intraparticle diffusivity values is $0.0044-$ $0.0052 \mathrm{~cm}^{2} / \mathrm{s}$ (see Table 4 ). Surface reaction rate constant and intraparticle diffusivity slightly decrease with hydrogen concentration. These facts may indicate that hydrogen affects both surface reaction of $\mathrm{H}_{2} \mathrm{~S}$ with the sorbent and intraparticle diffusivity of $\mathrm{H}_{2} \mathrm{~S}$ through the porous sorbent (see Figure 18).

\section{CONCLUSIONS}

The following conclusions were drawn based on the experimental data generated from the differential reactor system, and their interpretations,

- Initial absorption of $\mathrm{H}_{2} \mathrm{~S}$ into the AHI-5 sorbent is affected with absorption temperature for short absorption duration of $4-180$ seconds. The range of surface reaction rate constant is $0.16-1.2 \mathrm{~cm} / \mathrm{s}$. The range of intraparticle diffusivity values of the sorbent is $0.0002-$ $0.0029 \mathrm{~cm}^{2} / \mathrm{s}$. These observations may indicate that reactivity of the AHI-5 sorbent be dependent on reaction temperature in the temperature range of $350-500^{\circ} \mathrm{C}$. However, reactivity of the sorbent is much lower at $350^{\circ} \mathrm{C}$ than that at $500^{\circ} \mathrm{C}$. These facts also suggest that the controlling steps for the initial absorption of $\mathrm{H}_{2} \mathrm{~S}$ into sorbent particles are surface reaction of $\mathrm{H}_{2} \mathrm{~S}$ with reactive sites of sorbent particles and intraparticle diffusivity through pores of the sorbent. 
- The surface reaction rate constant and the intraparticle diffusivity value increases with the reaction temperatures up to $450^{\circ} \mathrm{C}$. However, the surface reaction rate constant increases steeply, and the intraparticle diffusivity value decreases in the temperature range of $475-$ $500^{\circ} \mathrm{C}$. These facts may indicate that collapsing the pore structure of the sorbent results in the decreased intraparticle diffusivity value in the temperature range of $475-500^{\circ} \mathrm{C}$.

- Amounts of the AHI-5 sorbent affect slightly initial dynamic absorption of $\mathrm{H}_{2} \mathrm{~S}$ into the sorbent in the sorbent amount range of $0.005-0.02 \mathrm{~g}$. The range of the surface reaction rate constant is $0.86-1.11 \mathrm{~cm} / \mathrm{s}$, and the range of the intraparticle diffusivity values is $0.0018-$ $0.0025 \mathrm{~cm}^{2} / \mathrm{s}$. Absorption of $\mathrm{H}_{2} \mathrm{~S}$ into the sorbent increases with decreased amount of the sorbent in the reactor. The surface reaction rate constant decrease with increased amount of the sorbent. The intraparticle diffusity values increase generally with increased amount of the sorbent. These observations may suggest that mass transfer of $\mathrm{H}_{2} \mathrm{~S}$ from the bulk gaseous phase to the surface of the solid sorbent affects somewhat the overall reaction of $\mathrm{H}_{2} \mathrm{~S}$ with the sorbent

- Concentrations of $\mathrm{H}_{2} \mathrm{~S}$ affect significantly initial dynamic absorption of $\mathrm{H}_{2} \mathrm{~S}$ into the AHI-5 sorbent in the $\mathrm{H}_{2} \mathrm{~S}$ concentration range of $9000-18000 \mathrm{ppm}$. The range of surface reaction rate constant is $0.94-1.38 \mathrm{~cm} / \mathrm{s}$, and the range of intraparticle diffusivity values is $0.0023-$ $0.0031 \mathrm{~cm}^{2} / \mathrm{s}$. The reaction rate constants of the sorbent decrease with increased $\mathrm{H}_{2} \mathrm{~S}$ concentration. The intraparticle diffusivity values of $\mathrm{H}_{2} \mathrm{~S}$ through the porous sorbent also decrease with increased $\mathrm{H}_{2} \mathrm{~S}$ concentrations in the $\mathrm{H}_{2} \mathrm{~S}$ concentration range of $9000-18000$ ppm.

- Initial absorption of $\mathrm{H}_{2} \mathrm{~S}$ into the AHI-5 sorbent is affected with concentrations of moisture for short absorption duration of 4-180 seconds. These facts may indicate that moisture is not inert for the reaction of $\mathrm{H}_{2} \mathrm{~S}$ with the AHI-5 sorbent. The range of surface reaction rate constants is $0.82-0.96 \mathrm{~cm} / \mathrm{s}$, and the range of intraparticle diffusivity values is $0.0019-$ $0.0025 \mathrm{~cm}^{2} / \mathrm{s}$. These observations may indicate that reactivity of the AHI- 5 sorbent and intraparticle diffusivity increase slightly with increased moisture concentrations.

- Concentrations of hydrogen affect slightly initial dynamic absorption of $\mathrm{H}_{2} \mathrm{~S}$ into the AHI-5 sorbent in the hydrogen concentration range of $5-27 \mathrm{v} \%$. The range of the initial surface reaction rate constant is $0.84-1.01 \mathrm{~cm} / \mathrm{s}$, and the range of the intraparticle diffusivity values is $0.0044-0.0052 \mathrm{~cm}^{2} / \mathrm{s}$. Surface reaction rate constant and intraparticle diffusivity slightly decrease with hydrogen concentration. These facts may indicate that hydrogen affects both surface reaction of $\mathrm{H}_{2} \mathrm{~S}$ with the sorbent and intraparticle diffusivity of $\mathrm{H}_{2} \mathrm{~S}$ through porous sorbents.

\section{REFERENCES}

1. Octave Levenspiel, Chemical Reaction Engineering, 3rd Edition, John Wiley \& Sons, 1999

2. Gilbert F. Froment, Chemical Reactor Analysis and Design, 2nd Edition, John Wiley \& Sons, 1990 
3. James J. Carberry, Chemical and Catalytic Reaction Engineering, McGraw-Hill, 1976

\section{PUBLICATIONS AND PRESENTATIONS}

"Reactivity of Metal Oxide Sorbents in the Removal of Hot Hydrogen Sulfide", published in Recent Developments in Air Pollution Control, edited by Mark P. Cal, Pg 77-87, Topical Conference Proceedings for the Spring AIChE National Meeting, March 5-9, 2000, Atlanta, GA.

"Reactivity of Metal Oxide Sorbents in the Removal of Hot Hydrogen Sulfide", presented at the Spring AIChE National Meeting, March 5-9, 2000, Atlanta, GA.

"Initial Reaction Kinetics of Solid Sorbents with $\mathrm{H}_{2} \mathrm{~S}$ at High Temperatures", presented at the AIChE Annual Meeting, November 12-17, 2000, Westin Bonaventure/Marriott Downtown Los Angeles, CA.

"Reactivity of Formulated Metal Oxide Sorbents with Hot Hydrogen Sulfide" (in press), Separation Science and Technology. 